\title{
2 \\ The effect of COVID-19 mRNA vaccine on respiratory system: human lung carcinoma cells by means of Raman spectroscopy and imaging
}

\section{H. Abramczyk ${ }^{* 1}$, J. Surmacki ${ }^{1}$}

$1 \quad$ Lodz University of Technology, Institute of Applied Radiation Chemistry, Laboratory of Laser Molecular Spectroscopy, Wróblewskiego 15, 93-590 Łódź, Poland

* Correspondence: halina.abramczyk@p.lodz.pl; Tel.: +48 4263131 88, H.A.

Abstract: The paper presents the effect of COVID-19 mRNA (Pfizer/BioNTech) vaccine on human lung carcinoma epithelial cells (A549) studied by means of Raman spectroscopy and imaging. The paper will also focus on Raman imaging as a tool to explore apoptosis and oxidative phosphorylation in mitochondrial dysfunctions. The presented Raman results show alterations in the reduction-oxidation pathways associated with cytochrome c. We found that the COVID-19 mRNA vaccine modulates the concentration of cytochrome $\mathrm{c}$ upon incubation with tumorous lung cells. Concentration of oxidized form of cytochrome $\mathrm{c}$ in mitochondria of lung cells has been shown to decrease upon incubation the mRNA vaccine. Lower concentration of oxidized cytochrome $\mathrm{c}$ in mitochondria indicates lower effectiveness of oxidative phosphorylation (respiration), reduced apoptosis and lessened ATP production. Moreover, mRNA vaccine increases significantly de novo lipids synthesis in lipid droplets and alterations in biochemical composition that suggest the increasing role of signaling. mRNA vaccine does not produce statistically significant changes in cell nucleus. The observed alterations in biochemical profiles upon incubation with mRNA vaccine in the specific organelles of the tumorous lung cells are similar to those we observe for other types of cancer, particularly brain glial cells.

Keywords: COVID-19 mRNA vaccines; SARS-Cov-2; cell lines of lung carcinoma; human lung cancer; Raman imaging; Raman spectroscopy, cytochrome c, mitochondrial dysfunction, apoptosis, oxidative phosphorylation

\section{Introduction}

The pandemic outbreak in 2019 generating acute respiratory syndrome caused 296496809 confirmed cases of COVID-19, including 5462631 deaths, reported to WHO [1]. In response to the urgent need for a vaccine, pharmaceutical companies including Pfizer/BioNTech in 2020 proposed vaccines based on mRNA technology. The Pfizer/BioNTech vaccine (BNT162b2) is more than 90\% effective against COVID-19 [2].

As of 7 January 2022, a total of 9.36 billion doses have been administered across 184 countries vaccine doses have been administered [3].

In order to enter the host cells, the virus uses the $S$ surface protein, the so-called spike protein (spike $S$ protein). Vaccines based on mRNA technology are designed to produce antibodies to the spike proteins. These proteins are designed to trigger the production of antibodies, which are then transferred to the host's immune system.

The pandemics has witnessed an explosion in research examining the interplay between the immune response and the intracellular metabolic pathways that mediate it. Research in the field of immunometabolism has revealed that similar mechanisms regulate the host response to infection, autoimmunity, and cancer. The new tools by Raman imaging we present in this paper raise exciting possibilities for new ways to understand pathways of our immune responses, recognize metabolites that regulates these pathways and 
suggest how we might use them to optimize vaccinations to stimulate the conditions of adaptive immune system.

We will show that the key molecule in immunometabolism is cytochrome c. Cytochrome $\mathrm{c}$ is a key protein that is needed to maintain life (respiration) and cell death (apoptosis). Life and death decisions are made not by cytochrome $c$ itself but rather by whatever causes its release from the mitochondria.

The down-regulation of cytochrome $\mathrm{c}$ responses in mitochondria after an incubation with mRNA vaccine has been presented. Dysregulation of apoptosis and oxidative phosphorylation in cells of the immune system can have severe consequences, which may result in diseases, including cancer and autoimmunity. This paper summarizes our current understanding of the role of cytochrome $\mathrm{c}$ in cancer and immune responses and aims to point out future directions of research.

In the paper, we present effect of COVID-19 mRNA (Pfizer/BioNTech) on the human carcinoma lung epithelial cells by using a novel non-invasive tool such as Raman imaging. Here we demonstrate that Raman imaging reveal new expanses on the role of basic mechanisms of cancer pathology and effect of mRNA vaccines. This approach can monitor interactions in tumor microenvironment and mechanisms related to immune response.

Raman spectroscopy and imaging enable quantitative and non-invasive monitoring of intracellular changes without the need of using external markers. Traditional methods of molecular biology require the destruction of cell membranes and the isolation of intracellular components to study the biochemical changes inside cells. In Raman imaging, we do not need to destroy cells to learn about biochemical composition of intracellular structures (cell organelles). Tracking alterations in biochemical composition in separate organelles is extremely valuable in establishing molecular mechanism of cancer development and mechanisms of infections. Until now, no technology has proven effective for detecting concentration of specific compounds in separate cell organelles of living cells. Therefore, existing analytical technologies cannot detect the full extent of biolocalization inside and outside specific organelles.

We will concentrate on human cancer lung cells upon incubation with mRNA vaccine. The reason is that cancer diseases are the most serious cause of death, exceeding heart disease, strokes, pneumonia and COVID-19. Although at the moment there is no vaccine against most cancers, rapid development of mRNA vaccines may help in development anticancer vaccines.

The announcement of effective and safe vaccines for COVID-19 has been greeted with enthusiasm. The vaccines currently used in the global vaccination campaign (9.36 billion doses have been administered across 184 countries, according to data collected [3].

While COVID-19 vaccines bring potential hope for a return to some kind of normality, many of the fundamental mechanisms by which mRNA vaccines induce strong responses are still incompletely understood and should be continued [2]. The mRNA vaccine encoding the COVID-19 spike (S) protein encapsulated in lipid nanoparticles gain entry into dendritic cells (DCs) at the injection site or within lymph nodes, resulting in production of high levels of $S$ protein.

Nevertheless, there is still much to learn, particularly for patients suffering from cancer. It is not clear which cell specific activation contributes the most to vaccine efficacy and what activation may inhibit the generation of adaptive immunity or lead to poor tolerability of the vaccine [4]. There are controversies on harmful effects from spike S protein produced by COVID-19 vaccination and long-term effects. Researchers have warned that COVID-19 mRNA (Pfizer/BioNTech) vaccine induces complex reprogramming of innate immune responses that should be considered in the development and use of mRNA-based vaccines [5]. There are also controversies on biodistribution of mRNA vaccines. It has been reported [6] that intramuscular vaccines (Pfizer/BioNTech) in macaques (a type of monkey) remain near the site of injection (the arm muscle) and local lymph nodes. The lymphatic system also remove waste materials. Similar results were obtained for a mRNA vaccine against $\mathrm{H} 10 \mathrm{~N} 8$ and H7N9 influenza viruses in mice [6]. However, recent results on interactions between the immune system and the viral proteins that induce immunity 
against COVID-19 suggest that the mechanisms may be more complex than previously thought [7]. Evidence has been found that spike S protein of COVID-19 has remain not only near the site of injection, but also circulate in the blood. COVID-19 proteins were measured in longitudinal plasma samples collected from 13 participants who received two doses of mRNA-1273 vaccine. 11 of 13 participants showed detectable levels of COVID-19 protein as early as day one upon first vaccine injection. Clearance of detectable COVID-19 protein is correlated with production of IgG and IgA [8].

In the view of the recent results it is important to monitor biodistribution and location of spike $S$ protein from mRNA vaccines and study the effects of the COVID-19 spike $S$ protein on human host cells in vitro and appropriate experimental animal models.

Visualization of alterations in single cells upon delivery of mRNA vaccines would help evaluate the efficacy of candidate formulations and aid their rational design for preclinical and translational studies. Here, we show that Raman imaging allows for quantitative, and non-invasive monitoring response to mRNA vaccine in specific organelles without any labeling.

In this paper we will study the effect for possible consequences of COVID-19 mRNA vaccine (Pfizer/BioNTech BNT162b2) on the human respiratory system. We have studied in detail the biochemical alterations in specific organelles of human lung epithelial cancer cells. Thus, we can compare the effect of mRNA on cancer lung cells with the effect of cancer itself. As far as we know the mRNA Pfizer vaccine has not been tested for patients suffering of cancer. Therefore, this contribution will help monitoring responses in host lung cells similar to a viral infection, because the incubation with COVID-19 mRNA vaccine mimics COVID-19 infection, but instead of the whole virus, only one key protein $S$ for the immune response is synthesized, without causing COVID-19.

We will study human lung cancer cells in vitro (A549) by Raman imaging. We will monitor the effect of the mRNA vaccine on biodistribution of different chemical components, particularly cytochrome c, in the specific organelles of a cell: nucleus, mitochondria, lipid droplets, cytoplasm and membrane.

In the presented study we will identify dynamics and biochemical composition of the organelles through characteristic Raman spectra upon injection of COVID-19 mRNA vaccine and incubation with the vaccine in vitro cells. We will show also that Raman spectroscopy and Raman imaging are competitive clinical diagnostics tools for cancer diseases linked to mitochondrial dysfunction and are a prerequisite for successful pharmacotherapy of cancer.

In this paper we explore alterations in reduction-oxidation pathways related to Cyt c in human cancer lung cells upon incubation in vitro with COVID-19 mRNA vaccine (Pfizer/BioNTech BNT162b2).

\section{Materials and Methods}

\subsection{Reagents}

Cytochrome c (C 2506) was purchased from Sigma Aldrich (Poland).

\subsection{In vitro cells culturing and incubation with vaccine}

Cell culture and preparation for Raman imaging

The studies were performed on a human lung carcinoma cell line A549 (ATCC CCL185) purchased from American Type Culture Collection (ATCC). The A549 cells were maintained in F-12K medium (ATCC 30-2004) supplemented with $10 \%$ fetal bovine serum (ATCC 30-2020) without antibiotics in a humidified incubator at $37^{\circ} \mathrm{C}$ and $5 \% \mathrm{CO}_{2}$ atmosphere. For Raman imaging cells were seeded on $\mathrm{CaF}_{2}$ window (Crystran Ltd, $\mathrm{CaF}_{2}$ Raman grade optically polished window $25 \mathrm{~mm}$ dia $\times 1 \mathrm{~mm}$ thick, no. CAFP25-1R) in $35 \mathrm{~mm}$ Petri dish at a density of $5 \times 10^{4}$ cells per Petri dish. On the following day culture medium was replaced with culture medium supplemented with 1 or $60 \mu \mathrm{L}$ COVID-19 mRNA vaccine Pfizer/BioNTech per $1 \mathrm{~mL}$ of medium. Cells were incubated with the vaccine for 1, 24, 72, and 96 hours. Before Raman examination, the growing medium was removed and the 
cells were fixed with 10\% formalin for 10 minutes and kept in phosphate-buffered saline (PBS, Gibco no. 10010023) during the experiment.

\subsection{Raman imaging and spectroscopy}

Raman spectroscopy is an analytical technique where inelastic scattered light is used to obtain the information about the vibrational energy of analyzed samples. In the vast majority of scattering events, the energy of the molecule is unchanged after its interaction with the photon; and therefore the wavelength, of the scattered photon is equal to that of the incident photon. This is called elastic (energy of scattering particle is preserved) or Rayleigh scattering and is the dominant process during interaction of photon with the molecule. In a much rarer event (approximately 1 in 10 million photons) Raman scattering occurs, which is an inelastic scattering process with a transfer of energy between the molecule and scattered photon. If the molecule gains energy from the photon during the scattering (excited to a higher vibrational level) then the scattered photon loses energy and its wavelength increases which is called Stokes Raman scattering. Inversely, if the molecule loses energy by relaxing to a lower vibrational level the scattered photon gains the corresponding energy and its wavelength decreases; which is called Anti-Stokes Raman scattering. Quantum-mechanically, Stokes and Anti-Stokes are equally probable processes. However, with an ensemble of molecules, the majority of molecules will be in the ground vibrational level (Boltzmann distribution) and Stokes scattering is statistically more probable process. In consequence, the Stokes Raman scattering is always more intense than the Anti-Stokes component and for this reason, it is nearly always the Stokes Raman scattering that is measured by Raman spectroscopy. Scheme 1 presents illustration of Rayleigh, Stokes and Anti-Stokes Raman Scattering.

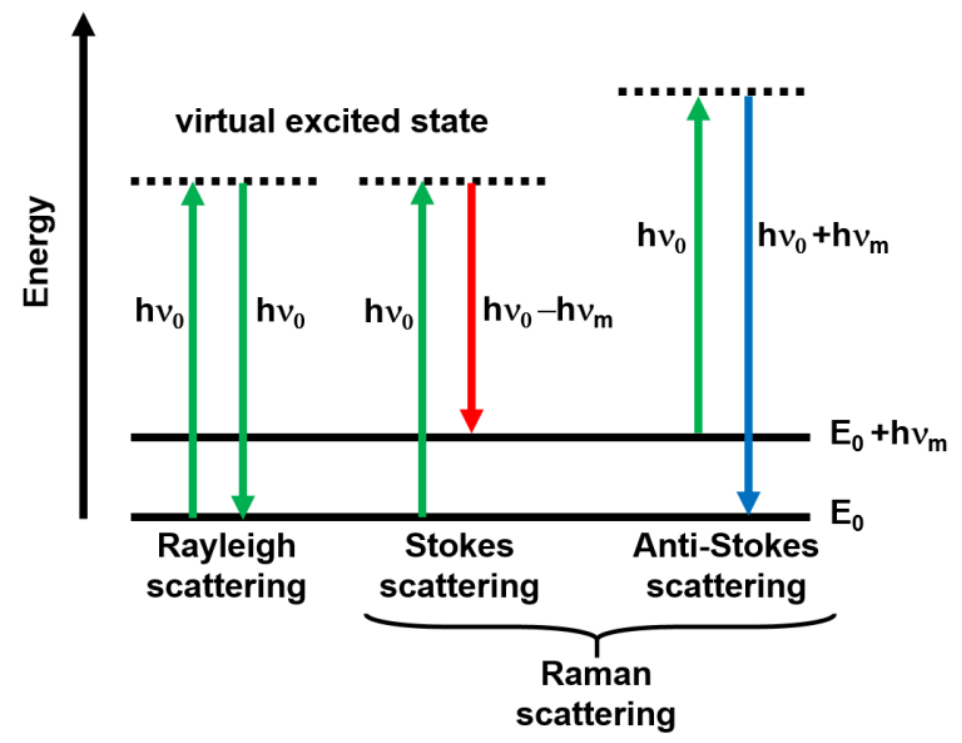

Scheme 1. Schematic presentation of scattering phenomena.

Raman imaging is a technique based on Raman scattering allowing not only a single spectrum acquisition characteristic for a single point of the sample but also the analysis of vibrational spectra of any sample area. The imaging mode allows the analysis of distribution of different chemical molecules inside the sample. Using algorithms such as Cluster Analysis (see section 2.4) based on 2D data obtained by using Raman imaging make possible to create Raman maps to visualize cell's substructures: nucleus, mitochondria, lipid structures, cytoplasm, cell membrane and learn about their biocomposition. 

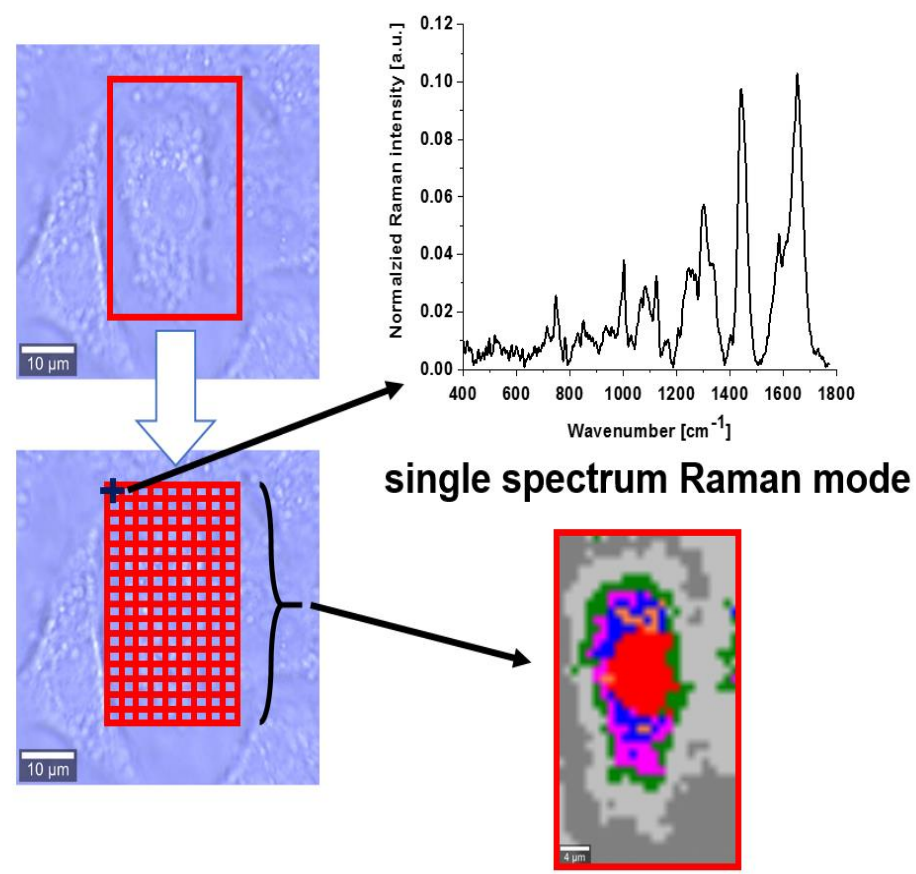

Raman imaging mode

Scheme 2. Schematic comparison of modes of data acquisition: Raman single spectra and Raman imaging of single cell (A549)

Raman spectra and images were recorded using a confocal Raman microscope (WITec (alpha 300 RSA+), Ulm, Germany) in the Laboratory of Laser Molecular Spectroscopy, Lodz University of Technology, Poland. The Raman microscope consists of an Olympus microscope (Olympus Düsseldorf, Germany), a UHTS (Ultra-High-Throughput Screening) monochromator (WITec, Ulm, Germany) and a thermoelectrically cooled CCD camera ANDOR Newton DU970N-UVB-353 (EMCCD (Electron Multiplying Charge Coupled Device, Andor Technology, Belfast, Northern Ireland) chip with $1600 \times 200$ pixel format, $16 \mu \mathrm{m}$ dimension each) at $-60^{\circ} \mathrm{C}$ with full vertical binning. A $40 \times$ water immersion objective (Zeiss, W Plan-Apochromat 40×/1.0 DIC M27 (FWD = $2.5 \mathrm{~mm}$ ), VIS-IR) was used for cell lines measurements. The excitation laser at $532 \mathrm{~nm}$ was focused on the sample to the laser spot of $1 \mu \mathrm{m}$ and was coupled to the microscope via an optical fiber with a diameter of $50 \mu \mathrm{m}$. The average laser excitation power was $10 \mathrm{~mW}$, and the collection time was 0.5 and $1 \mathrm{~s}$ for Raman images. Raman images were recorded with a spatial resolution of $1 \times 1 \mu \mathrm{m}$. The Raman spectrometer was calibrated every day prior to the measurements using a silica plate with a maximum peak at $520.7 \mathrm{~cm}^{-1}$.

\subsection{Data processing}

Data acquisition and processing were performed using WITec Project Plus software. The background subtraction and the normalization (model: divided by norm (divide the spectrum by the dataset norm)) were performed by using Origin software. The normalization model: divided by norm was performed according to the formula:

where:

$$
\begin{gathered}
V^{\prime}=\frac{V}{\|V\|} \\
\|V\|=\sqrt{v_{1}^{2}+v_{2}^{2}+\cdots v_{n}^{2}}
\end{gathered}
$$

$v_{n}$ is the $\mathrm{n}^{\text {th }} \mathrm{V}$ values.

The normalization was performed for low $\left(370-1770 \mathrm{~cm}^{-1}\right)$ and high $\left(2700-3100 \mathrm{~cm}^{-1}\right)$ frequency spectral regions separately. 


\subsection{Cluster analysis}

Spectroscopic data were analyzed using Cluster Analysis method. Briefly, Cluster Analysis is a form of exploratory data analysis in which observations are divided into different groups that have some common characteristics - vibrational features in our case. Cluster Analysis constructs groups (or classes or clusters) based on the principle that within a group of observations (the Raman spectra) must be as similar as possible, while the observations (the Raman spectra) belonging to different groups must be different.

The partition of $n$ observations $(x)$ into $k(k \leq n)$ clusters $S$ should be done to minimize the variance (Var) according to the formula:

$$
\arg \min _{S} \sum_{i=1}^{k} \sum_{x \in S i}\left\|x \mu_{i}\right\|^{2}=\arg \min _{S} \sum_{i=1}^{k}\left|S_{i}\right| \operatorname{Var} S_{i} \mid
$$

where

$\mu_{i}$ is the mean of points $S_{i}$.

The Raman maps presented in the manuscript were constructed based on the principles of Cluster Analysis described above. The number of clusters was 7 (the number of clusters characterized by different average Raman spectra), which describe the inhomogeneous distribution of chemical components within the cell).

\section{Results}

To learn about alterations in biochemical composition in cell organelles by methods of conventional molecular biology one have to disrupt a cell to break it to open and release the cellular components in specific organelles. Using Raman imaging we do not need to disrupt cells to learn about the localization, distribution and biochemical composition of specific compounds in different organelles.

To properly address alterations in single lung cells upon incubation with COVID-19 mRNA vaccine, we systematically investigated how Raman spectroscopy and Raman imaging monitor responses to the vaccine in specific organelles.

Figure. 1 shows the Raman image of a single cell of highly aggressive lung cancer incubated with mRNA-based Pfizer/BioNTech vaccine (dose $60 \mu \mathrm{L}$ ) for 96 hours and corresponding Raman imagines of specific organelles. The Raman images were created by Kmeans cluster analysis using 7 clusters. The blue color represents lipids in lipid droplets and rough endoplasmic reticulum, the orange color represents lipid droplets (filled with triacylglycerols of monounsaturated type (TAG)), magenta color represents mitochondria, red color represents nucleus, green - cytoplasm, and light grey - membrane (the dark grey color corresponds to cell environment). 

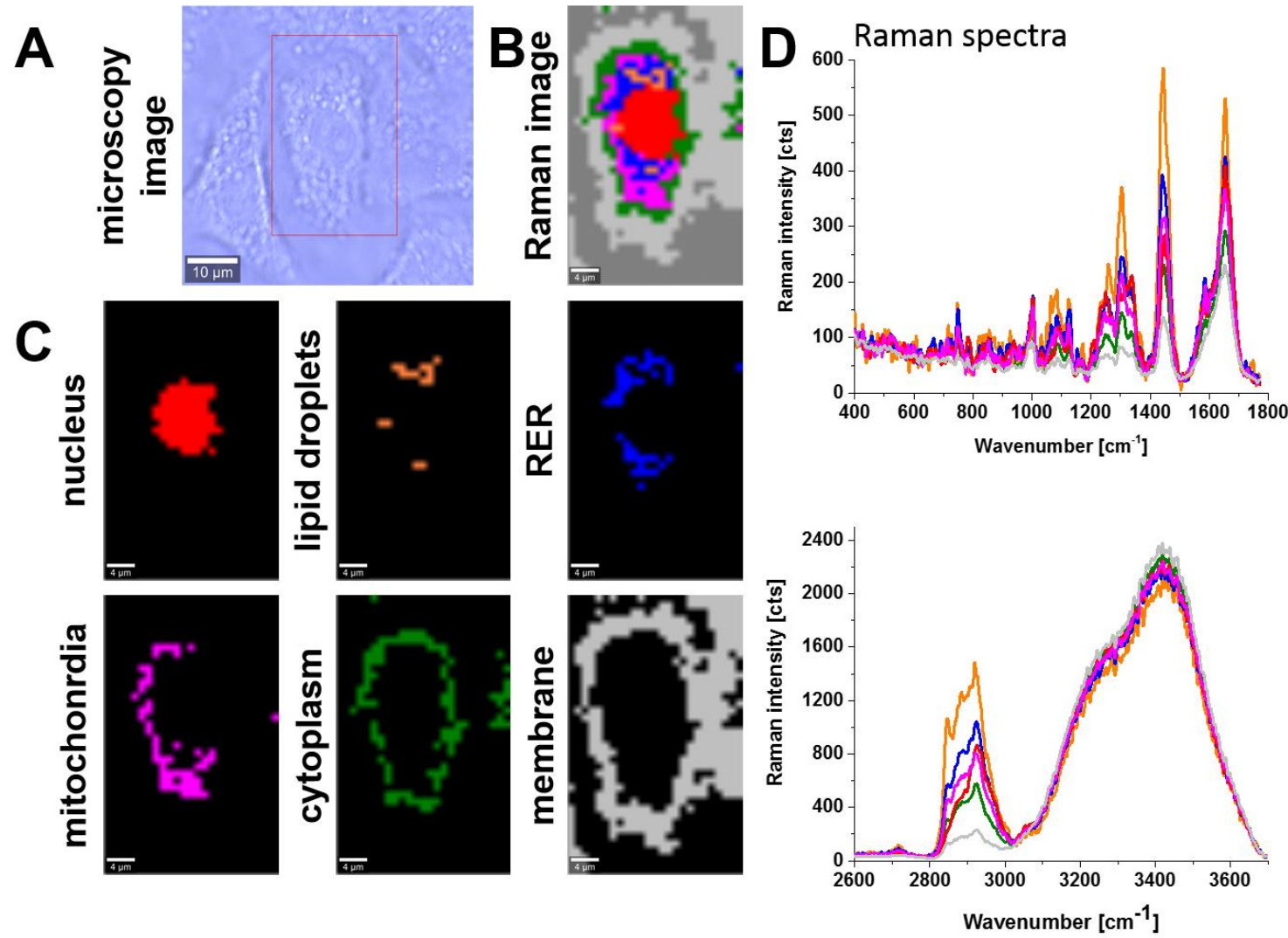

Figure 1. Microscopy image (A), Raman image of human lung carcinoma (A549) cell (25x40 $\mu \mathrm{m}$, resolution $1.0 \mu \mathrm{m})$ incubated with COVID-19 mRNA vaccine (dose $60 \mu \mathrm{L}$ ) for 96 hours (B) and Raman imagines of specific organelles: nucleus (red), lipid droplets (orange), rough endoplasmic reticulum (RER, blue), mitochondria (magenta), cytoplasm (green) and membrane (light grey) (C) with corresponding Raman spectra (D) at $532 \mathrm{~nm}$.

To properly address alterations in human lung cancer cells upon incubation with COVID-19 vaccine (Pfizer/BioNTech) we systematically investigated how the Raman imaging and spectroscopy monitor response of cells incubated with mRNA and control cells without mRNA incubation. 

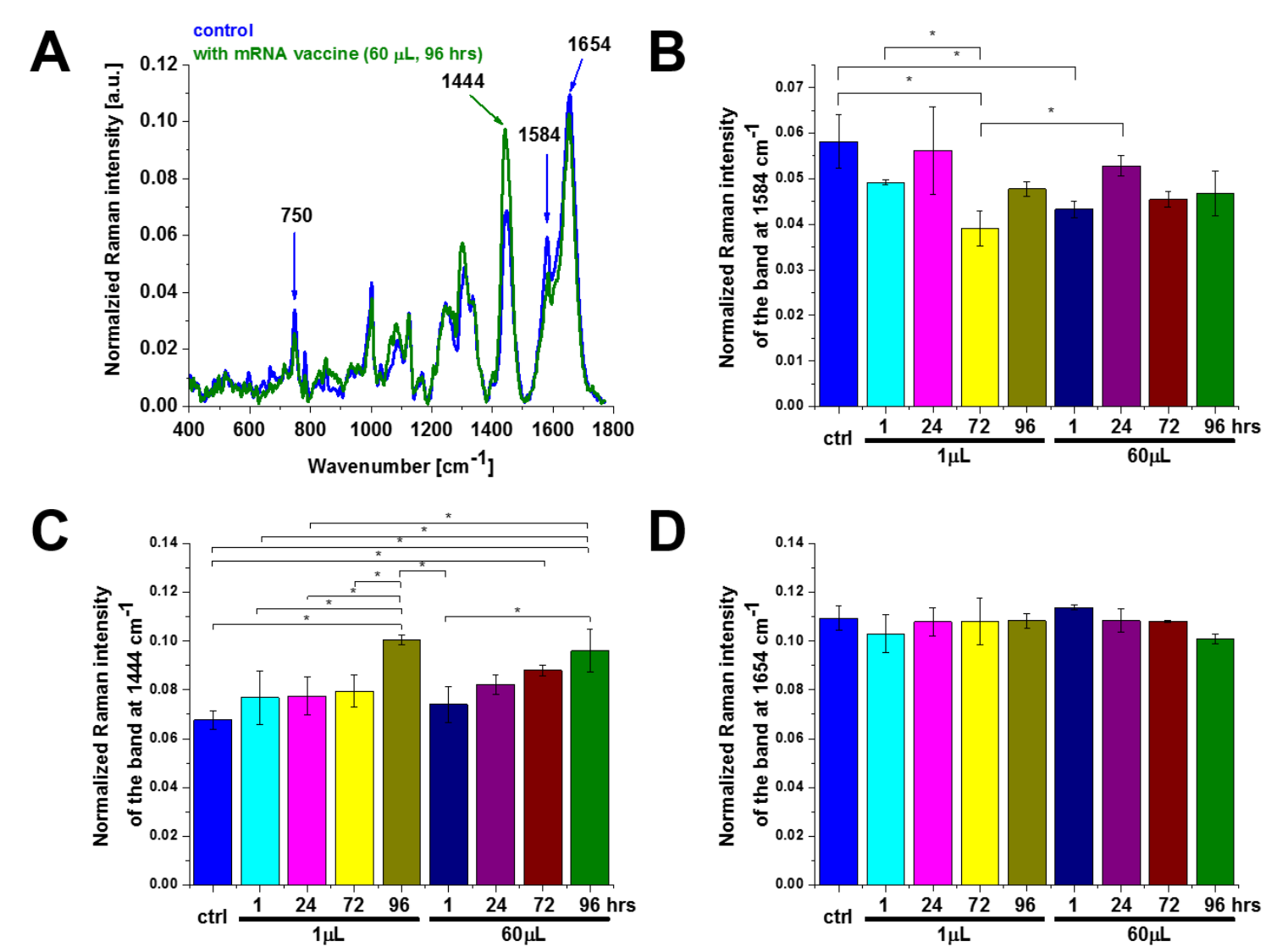

Figure 2. The effect of the COVID-19 mRNA vaccine on mitochondria in human lung carcinoma (A549) cell upon incubation for 1, 24, 72 and 96 hours for $1 \mu \mathrm{L}$ and $60 \mu \mathrm{L}$ doses at 1584, 1444 and $1654 \mathrm{~cm}^{-1}$ (number of cells: 3, number of Raman spectra for each single cell: minimum 1600, excitation wavelength: $532 \mathrm{~nm}$, laser power: $10 \mathrm{~mW}$, integration time: $1.0 \mathrm{sec}$ ). The one-way ANOVA using the Tukey test was used to calculate the value significance, asterisk * denotes that the differences are statistically significant, p-value $\leq 0.05$.

Detailed inspection into Fig. 2 demonstrates that the most significant changes occur at $750 \mathrm{~cm}^{-1}$ and $1584 \mathrm{~cm}^{-1}$ corresponding to cytochrome c [9] and at $1444 \mathrm{~cm}^{-1}$ corresponding to saturated $\mathrm{C}-\mathrm{H}$ vibrations of lipids [10]. Let us first concentrate on cytochrome c.

Briefly, cytochromes are classified on the basis of their lowest electronic energy absorption band in their reduced state. Therefore, we can distinguish cytochrome P450 (450 $\mathrm{nm})$, cytochrome c $(550 \mathrm{~nm})$, cytochromes b $(\approx 565 \mathrm{~nm})$, cytochromes a $(605 \mathrm{~nm})$. Due to the optical properties the laser excitation with the wavelength at $532 \mathrm{~nm}$, whose energy is in coincidence with the electron absorption of the $\mathrm{Q}$ band, leads to greatly enhanced intensity of the Raman scattering (called Resonance Raman effect), which facilitates the study of cytochrome c present at low concentrations under physiological conditions of humans. The cytochrome $\mathrm{c}$ is localized in mitochondrium at the internal mitochondrial membranes between the complex known as complex III (sometimes called also Coenzyme $\mathrm{Q}$ - Cyt C reductase or cytochrome bc1 complex) and the complex IV and play a key role in the electron transport chain of the oxidative phosphorylation (respiration) process. The oxidized cytochrome $\mathrm{c}\left(\mathrm{cyt} \mathrm{c} \mathrm{Fe}{ }^{3+}\right)$ is reduced to cytochrome c $\left(\mathrm{cyt} \mathrm{c} \mathrm{Fe}^{2+}\right.$ ) by the electron obtained from the complex III. The reduced cytochrome c passes an electron to the copper binuclear center of the complex IV, being oxidized back to cytochrome c (cyt c Fe ${ }^{3+}$ ). Complex IV, which contains two cytochromes a and a3 and two copper centers, is the final enzyme of the electron transport system. Therefore, under normal conditions cytochrome $\mathrm{c}$ in mitochondrium exists in the oxidized form. Under pathological conditions metabolic processes in mitochondrium results in down-regulation of the transfer between cyto- 
chrome $\mathrm{c}$ and the complex IV leading to increase of the reduced form of cytochrome. Raman signals of the reduced form are significantly higher than those of the oxidized form of cytochrome c [11]. Therefore, Raman spectra are excellent markers of redox status of cytochrome c.

The peak at $1584 \mathrm{~cm}^{-1}$ in Fig. 2 represents the "redox state Raman marker" of cytochrome c. Recently we demonstrated that this Raman vibration can serve as a sensitive indicator of oxidized and reduced forms of cytochrome c [9]. It indicates that the Raman peak at $1584 \mathrm{~cm}^{-1}$ can be used as a marker to explore apoptosis and oxidative phosphorylation in mitochondria $[16,18]$. This band reflects the dual face of cytochrome $\mathrm{c}$ in life and death decisions: apoptosis and oxidative phosphorylation. The balance between cancer cells proliferation (oxidative phosphorylation) and death (apoptosis) decide about level of cancer development $[12,13]$. The Raman signal of a single cell at $1584 \mathrm{~cm}^{-1}$ depends on cytochrome c concentration (which also depends on number of mitochondria in a cell), and the redox state (oxidized or reduced forms). The Raman intensity of the oxidized form is much smaller than that of the reduced form $[11,12,14,15]$.

Inside a normal mitochondrium cytochrome c exists in the oxidized form. Dysfunction of mitochondrium associated with several malignancies, including cancer or virus infections blocks the transfer of electrons between complexes III and IV of the respiratory chain, resulting in reducing the efficiency of the oxidative phosphorylation (respiration) process and lower ATP synthesis. This results in change of the redox status of cytochrome c to reduced form (cyt c Fe ${ }^{2+}$ ) [11]. Cytochrome c acts not only as mitochondrial redox carrier that transfers electrons between the membrane-embedded complexes III and IV, but also serves as a cytoplasmic apoptosis-triggering agent, activating the caspase cascade, when cytochrome $c$ is released from the intermembrane space of mitochondrium. The released from mitochondrium cytochrome $c$ interacts with apoptosis-protease activating factor 1 (Apaf-1) to trigger the caspase cascade in the cell and elicts an inflammatory response in the immune system. Activated caspases ultimately lead to apoptotic cell dismantling. Therefore, cytochrome c plays the role of a universal damage-associated molecular patterns (DAMPs) able of alarming the immune system for danger in any type of cell or tissue. DAMPs that are released from damaged or dying cells and activate the innate immune system by interacting with pattern recognition receptors (PRRs). Although DAMPs contribute to the host's defense, they also promote pathological inflammatory responses. Induction of cell death by apoptosis is indispensable for the normal functioning of the immune system [16] as well as in natural mechanisms protecting against cancer. When less cytochrome $\mathrm{c}$ is released as DAMP from damaged or dying cells the innate immune system is not activated to the sufficient level by interacting with pattern recognition receptors (PRRs).

The similar mechanism may play a key role in adaptive immune system, because it is reported that cytokines play an important role in adaptive immunity $[17,18]$. Depending on the cytokines they synthesize and secrete, $\mathrm{CD}^{+}$helper $\mathrm{T}$ cells (Th) can be divided into two subgroups, type I helper T lymphocytes (Thl) and type II helper T cells (Th2) [17].

We provided evidence that the balance between apoptosis and the oxidative phosphorylation is regulated by cardiolipin [11]. Cardiolipin-bound Cyt c, probably does not participate in electron shuttling of the respiratory chain [19] leading to production of reduced form of cytochrome $\mathrm{c}$ resulting in lower efficiency of respiration (oxidative phosphorylation) and lessened ATP production. The reduced form of cytochrome bound to cardiolipine cannot induce caspase and apoptosis process [11,19].

The intensity of the Raman signal at $1584 \mathrm{~cm}^{-1}$ corresponding to the concentration of cytochrome $\mathrm{c}$ in mitochondria depends on three factors: 1) redox status (oxidized or reduced form of cytochrome c, 2) number of cytochrome c molecules in mitochondria that depends on the number of mitochondria in a single cells, 3) release of cytochrome $c$ to cytoplasm. As the Raman signals of the reduced form are significantly higher than those of the oxidized form of cytochrome c [11] one can state that the Raman intensity of the band at $1584 \mathrm{~cm}^{-1}$ in Fig. 2 represents an oxidized form of cytochrome (cyt c Fe $\left.{ }^{3+}\right)$ both for the control and the cells upon incubation with the COVID-19 mRNA vaccine. In the next 
paragraph we demonstrated that there is no release of cytochrome c to cytoplasm. It indicates that the concentration of cytochrome $\mathrm{c}\left(\mathrm{cyt} \mathrm{c} \mathrm{Fe}^{3+}\right)$ is determined by the factor 2): the number of mitochondria in a single cell. The results on Fig. 2 show that the number of mitochondria in a single cell decrease upon incubation with mRNA determining their metabolic activities. The population of all the mitochondria of a given cell constitutes the chondriome. The number of mitochondria present in a cell depends upon the metabolic requirements of that cell, and may range from a few mitochondria to thousands of the organelles [20].

In the view of the results presented in Fig. 2 one can state that mRNA vaccine does not block the transfer of electrons between complexes III and IV of the respiratory chain, but decrease the number of mitochondria in cells which demonstrate lower metabolic requirements and lower efficiency of oxidative phosphorylation (respiration) and ATP synthesis.

It is worth of noticing that a similar effect of lower concentration of cytochrome c we observed in brain cancer cells. We showed that the Raman intensity of the band at 1584 $\mathrm{cm}^{-1}$ corresponding to the concentration of cytochrome $\mathrm{c}$ in mitochondria of a single cell in vitro decreases with brain tumor aggressiveness $[9,14]$.

\subsection{Cytoplasm-mRNA}

In order to check if the observed lower concentration of cytochrome $\mathrm{c}$ in mitochondria is related to release of cytochrome c (apoptosis) to cytoplasm we studied the localization of cytochrome c in cytoplasm.

Figure 3 shows the effect of the COVID-19 mRNA vaccine on the Raman spectra in cytoplasm of human lung carcinoma (A549) by Raman imaging compared with the control cells. Fig 3 shows a comparison of the average Raman for cytoplasm at $532 \mathrm{~nm}$ excitation with and without COVID-19 mRNA vaccine. One can see that that the Raman signal at $1584 \mathrm{~cm}^{-1}$ in cytoplasm is the same for control cells and those incubated with mRNA within statistical significance at $\mathrm{p}$-value $\leq 0.05$. It means that the lower concentration of cytochrome $\mathrm{c}$ in mitochondria is not related to the release to cytoplasm. In the view of this result from Fig. 3 one can state that the mRNA does not activate additional apoptosis via cytochrome $\mathrm{c}$ release and does not act as a cytoplasmic apoptosis-triggering agent. 

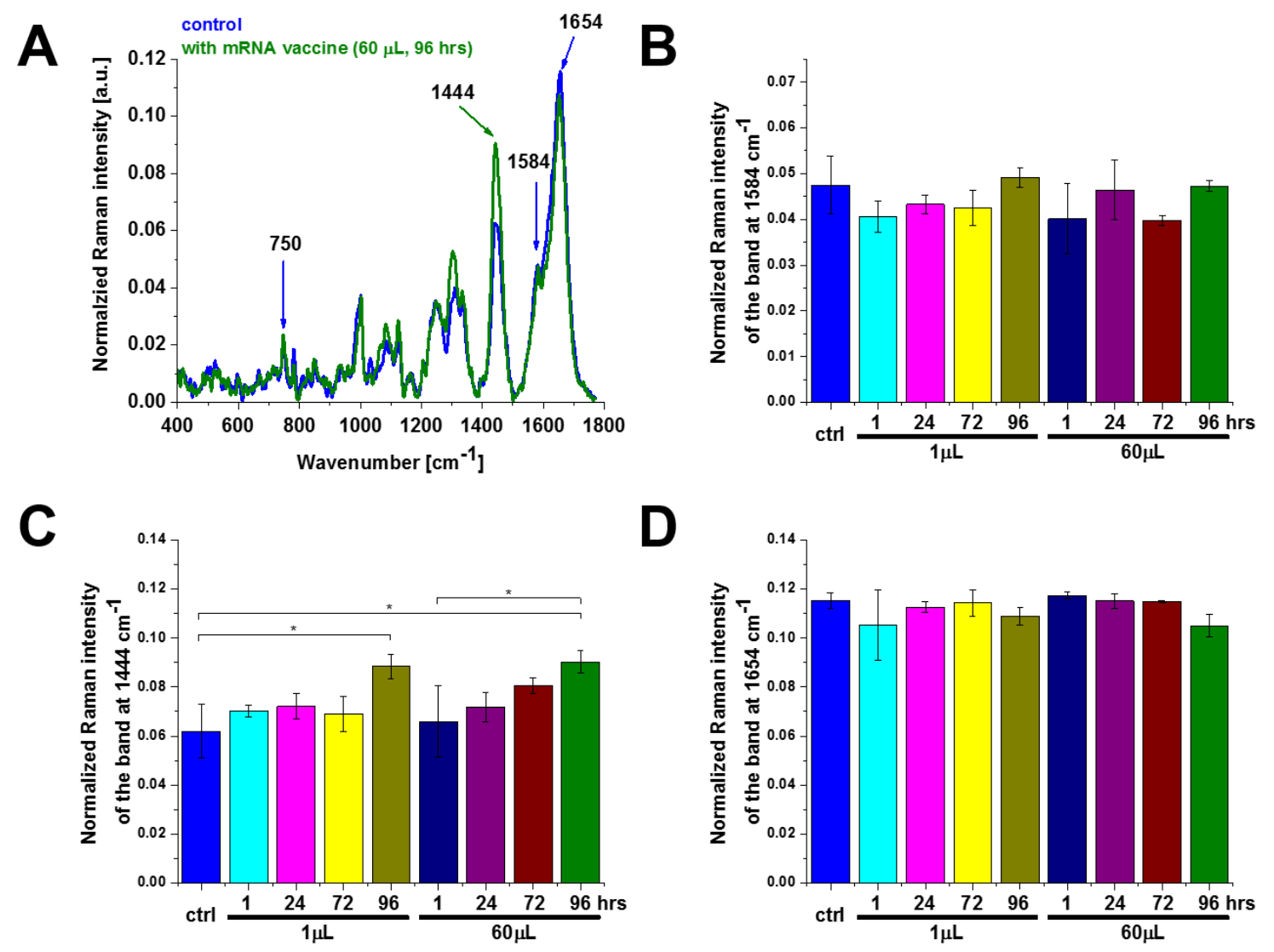

Figure 3. The effect of the COVID-19 mRNA vaccine on cytoplasm in human lung carcinoma (A549) cell upon incubation for 1, 24, 72 and 96 hours for $1 \mu \mathrm{L}$ and $60 \mu \mathrm{L}$ doses (number of cells: 3, number of Raman spectra for each single cell: minimum 1600, excitation wavelength: $532 \mathrm{~nm}$, laser power: $10 \mathrm{~mW}$, integration time: $1.0 \mathrm{sec}$ ). The one-way ANOVA using the Tukey test was used to calculate the value significance, asterisk * denotes that the differences are statistically significant, p-value $\leq 0.05$.

\subsection{Lipid droples-mRNA and rough endoplasmic reticulum-mRNA}

The significant changes in cytochrome c concentration occurs not only for mitochondria. Similar alterations in biochemical composition of cytochrome c reflected by the band at $1584 \mathrm{~cm}^{-1}$ are also observed in lipid droplets and in lipid structures of endoplasmic reticulum (ER) presented in Figures 4 and 5. Rough ER is studded with ribosomes that perform biological protein synthesis (mRNA translation). Ribosomes are the sites of protein synthesis for mRNA vaccines. Ribosomes are too small to be seen by resolution of Raman imaging.

Now we would like to concentrate on lipid synthesis upon mRNA vaccine. Figures 2-5 show that the Raman band at $1444 \mathrm{~cm}^{-1}$ along with cytochrome c is modified significantly upon incubation with mRNA. Indeed, the Raman signal at $1444 \mathrm{~cm}^{-1}$ corresponding to $\mathrm{C}-\mathrm{H}$ bending vibrations of lipids in lipid droplets (orange color in Fig.1) and in rough endoplasmic reticulum (blue color in Fig.1) increases significantly upon incubation with COVID-19 mRNA vaccine. The results indicates that mRNA reprograms lipid metabolism by up-regulation de novo lipid synthesis [21]. Recently we showed that the lipid droplets in cancer cells are predominantly filled with TAGs and are involved in energy storage. The lipid droplets in normal cells NHA are filled mainly with retinyl esters / retinol and are involved in signaling, especially JAK2/STAT6 pathway signaling [22]. The results presented in Figure 4 suggest that upon incubation with mRNA the role of signaling increases. Our results support those reported recently that COVID-19 spike protein elicits cell signaling in human host cells, which may have serious implications for possible consequences of COVID-19 vaccines [23]. 

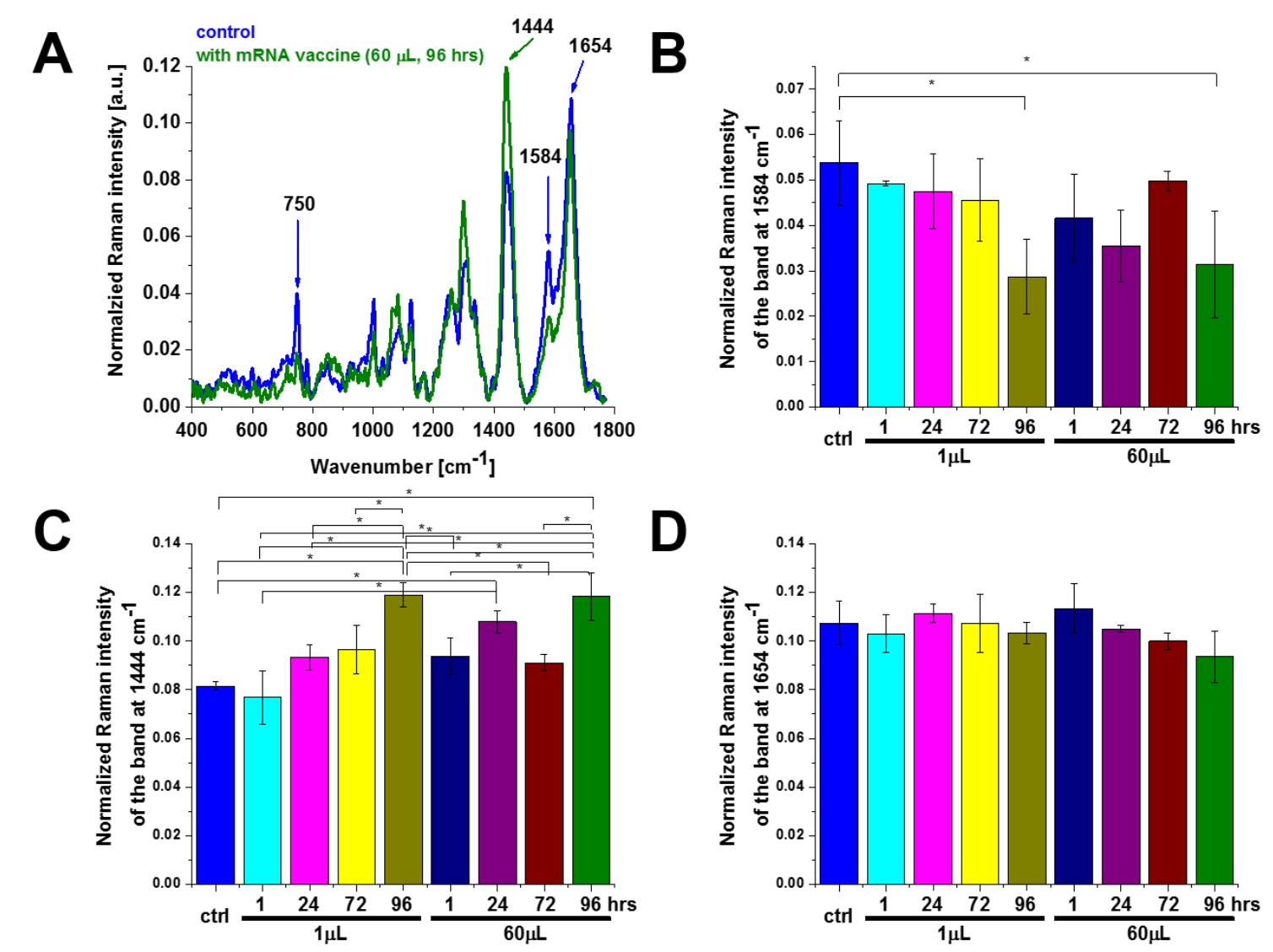

Figure 4. The effect of the COVID-19 mRNA vaccine on lipid droplets in human lung carcinoma (A549) cell upon incubation for 1, 24, 72 and 96 hours for $1 \mu \mathrm{L}$ and $60 \mu \mathrm{L}$ doses (number of cells: 3, number of Raman spectra for each single cell: minimum 1600, excitation wavelength: $532 \mathrm{~nm}$, laser power: $10 \mathrm{~mW}$, integration time: $1.0 \mathrm{sec}$ ). The one-way ANOVA using the Tukey test was used to calculate the value significance, asterisk * denotes that the differences are statistically significant, p-value $\leq 0.05$. 

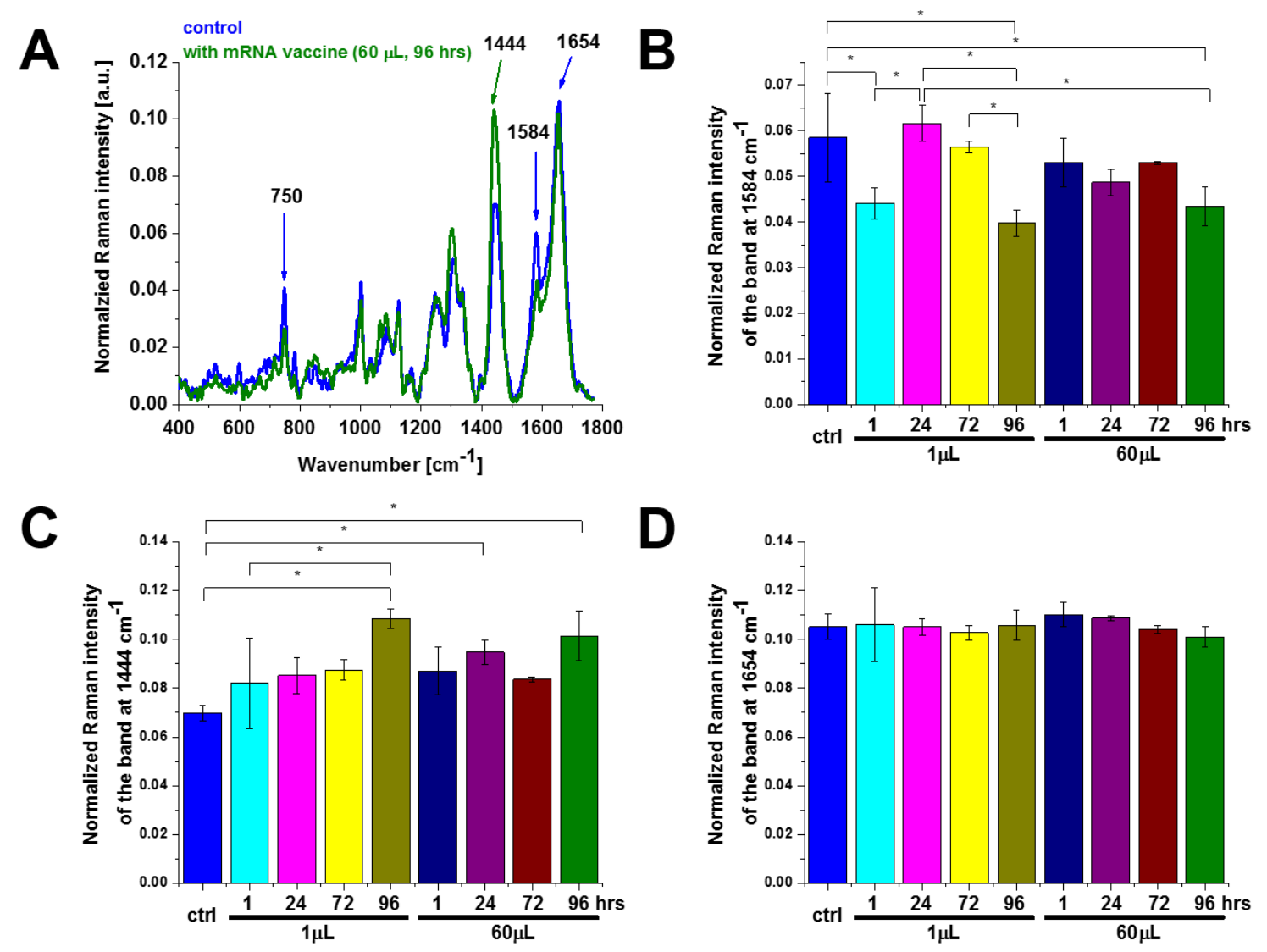

Figure 5. The effect of the COVID-19 mRNA vaccine on rough endoplasmic reticulum in human lung carcinoma (A549) cell upon incubation for 1, 24, 72 and 96 hours for $1 \mu \mathrm{L}$ and $60 \mu \mathrm{L}$ doses (number of cells: 3, number of Raman spectra for each single cell: minimum 1600, excitation wavelength: $532 \mathrm{~nm}$, laser power: $10 \mathrm{~mW}$, integration time: $1.0 \mathrm{sec}$ ). The oneway ANOVA using the Tukey test was used to calculate the value significance, asterisk * denotes that the differences are statistically significant, $\mathrm{p}$-value $\leq 0.05$.

\subsection{Nucleus-mRNA}

Most scientists claim that mRNA vaccine never enters the nucleus of the cell where our DNA (genetic material) is kept [4]. The cell breaks down and gets rid of the mRNA soon after it finished translation procedure. It is assumed that it is unplausible that an RNA vaccine would change our DNA, for many reasons. First, neither the coronavirus nor the RNA vaccines (which only code for the spike protein) have a reverse transcriptase. Therefore, RNA vaccines cannot produce DNA molecules. Another reason is that the cells keep their compartments well separated, and messenger RNAs cannot travel from the cytoplasm to the nucleus. Also, mRNAs are short-lived molecules. The vaccine's messenger does not stay inside the cell indefinitely, but is degraded after a few hours, without leaving a trace. Finally, clinical studies on thousands of people who have received the RNA vaccines have shown no sign of DNA modification so far. This is perhaps the best indication that these vaccines do not alter our genome. Therefore, is particularly important to monitor alterations in nucleus upon incubation with mRNA by Raman imaging. Figure 7 shows results obtained for nucleus without and upon incubation with vaccine by Raman imaging. Our results seem to support the conclusions that mRNA vaccine does not enter the DNA of the cell. 

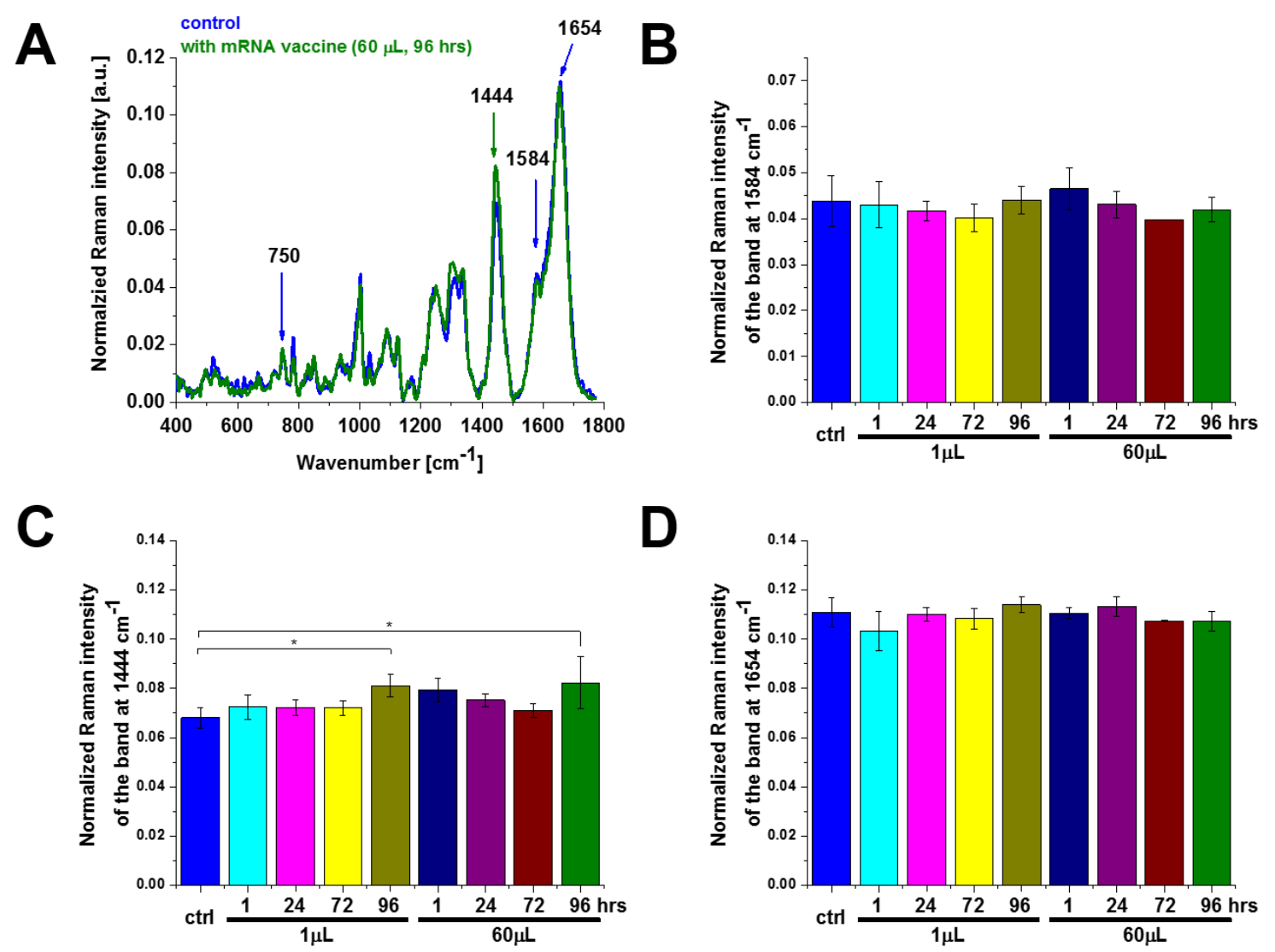

Figure 7. The effect of the COVID-19 mRNA vaccine on nucleus in human lung carcinoma (A549) cell upon incubation for 1, 24, 72 and 96 hours for $1 \mu \mathrm{L}$ and $60 \mu \mathrm{L}$ doses (number of cells: 3, number of Raman spectra for each single cell: minimum 1600, excitation wavelength: $532 \mathrm{~nm}$, laser power: $10 \mathrm{~mW}$, integration time: $1.0 \mathrm{sec}$ ). The one-way ANOVA using the Tukey test was used to calculate the value significance, asterisk * denotes that the differences are statistically significant, p-value $\leq 0.05$.

\subsection{Membrane-mRNA}

As it is well known professional antigen-presenting cells (APCs) play a crucial role in initiating immune responses. Under pathological conditions also epithelial cells act as nonprofessional APCs, thereby regulating immune responses at the site of exposure. Therefore, it is interesting to monitor alterations at the surface of the cell membranes upon incubation with mRNA.

Figure. 8 shows effect of incubation with COVID-19 mRNA vaccine compared to the control cells without mRNA at surface of the membrane. One can see that cytochrome $c$ activity at $1584 \mathrm{~cm}^{-1}$ does not change with statistical significance at $\mathrm{p}$-value $\leq 0.05$. 

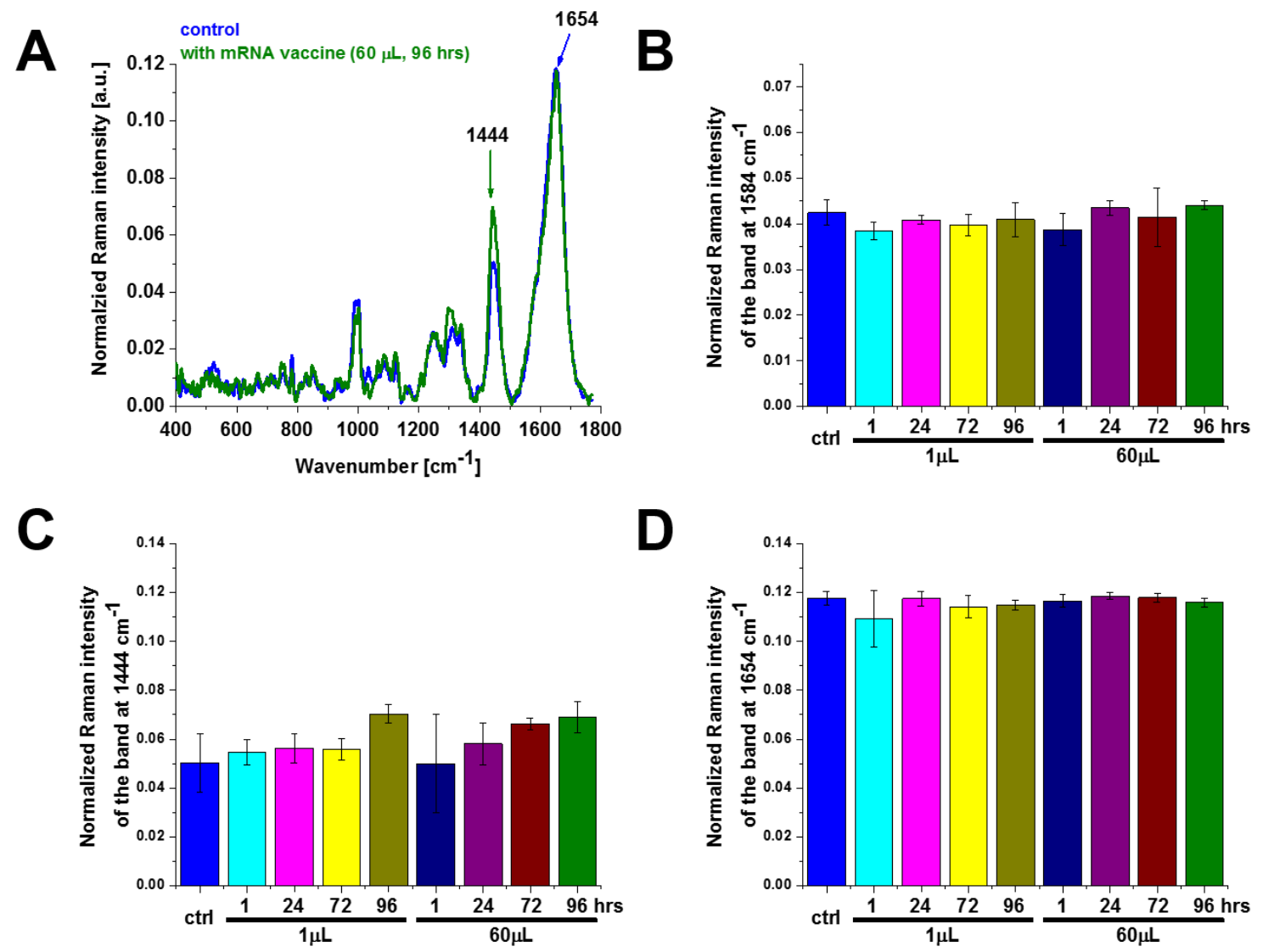

Figure 8. The effect of the COVID-19 mRNA vaccine on membrane in human lung carcinoma (A549) cell upon incubation for 1, 24, 72 and 96 hours for $1 \mu \mathrm{L}$ and $60 \mu \mathrm{L}$ doses (number of cells: 3, number of Raman spectra for each single cell: minimum 1600, excitation wavelength: $532 \mathrm{~nm}$, laser power: $10 \mathrm{~mW}$, integration time: $1.0 \mathrm{sec}$ ). The one-way ANOVA using the Tukey test was used to calculate the value significance, asterisk * denotes that the differences are statistically significant, p-value $\leq 0.05$.

\section{Conclusions}

We observed the effect of the COVID-19 mRNA vaccine on biodistribution of different chemical components, particularly cytochrome $c$, in the specific organelles of human lung cancer cells: nucleus, mitochondria, lipid droplets, cytoplasm and membrane.

Cytochrome c activity, which is responsible for apoptosis and oxidative phosphorylation, has been shown to be down-regulated by the COVID-19 mRNA vaccine (Pfizer/BioNTech). Lower concentration of oxidized cytochrome c observed in mitochondria in human lung cancer cells upon incubation with COVID-19 mRNA vaccine leads to reduced oxidative phosphorylation (respiration), and lessened ATP production. mRNA vaccine increases significantly de novo lipids synthesis in lipid droplets and rough endoplasmic reticulum. mRNA vaccine does not produce statistically significant changes in cell's nucleus. All observations may suggest that the COVID-19 mRNA vaccine Pfizer/BioNT vaccine reprograms cytochrome $\mathrm{c}$ and lipids in a similar way that is observed for cancers, which have been reported in our previous papers.

Author Contributions: Conceptualization - H.A.; investigation - J.S.; visualization - J.S. and H.A.; writing - original draft - H.A.; writing - review and editing - J.S. and H.A. All authors have read and agreed to the published version of the manuscript.

Funding: This work was supported by Statutory activity 2021: 501/3-34-1-1.

Institutional Review Board Statement: Not applicable.

Informed Consent Statement: Not applicable. 
Data Availability Statement: The raw data underlying the results presented in the study are available from Lodz University of Technology Institutional Data Access. Request for access to those data should be addressed to the Head of Laboratory of Laser Molecular Spectroscopy, Institute of Applied Radiation Chemistry, Lodz University of Technology. Data requests might be sent by email to the secretary of the Institute of Applied Radiation Chemistry: mitr@mitr.p.lodz.pl.

Conflicts of Interest: The authors declare no conflict of interest. The funders had no role in the design of the study; in the collection, analyses, or interpretation of data; in the writing of the manuscript, or in the decision to publish the results.

\section{References}

1. WHO Coronavirus (COVID-19) Dashboard I WHO Coronavirus (COVID-19) Dashboard With Vaccination Data Available online: https://covid19.who.int/ (accessed on 12 October 2021).

2. Doshi, P. Covid-19 Vaccines: In the Rush for Regulatory Approval, Do We Need More Data? The BMJ 2021, 373, 2020-2022, doi:10.1136/bmj.n1244.

3. More Than 9.6 Billion Shots Given: Covid-19 Tracker. Bloomberg.com.

4. Bettini, E.; Locci, M. SARS-CoV-2 MRNA Vaccines: Immunological Mechanism and Beyond. Vaccines 2021, 9, 147, doi:10.3390/VACCINES9020147.

5. Föhse, F.K.; Geckin, B.; Overheul, G.J.; van de Maat, J.; Kilic, G.; Bulut, O.; Dijkstra, H.; Lemmers, H.; Sarlea, S.A.; Reijnders, M.; et al. The BNT162b2 MRNA Vaccine Against SARS-CoV-2 Reprograms Both Adaptive and Innate Immune Responses; Social Science Research Network: Rochester, NY, 2021;

6. Ols, S.; Yang, L.; Thompson, E.A.; Pushparaj, P.; Tran, K.; Liang, F.; Lin, A.; Eriksson, B.; Karlsson Hedestam, G.B.; Wyatt, R.T.; et al. Route of Vaccine Administration Alters Antigen Trafficking but Not Innate or Adaptive Immunity. Cell Reports 2020, 30, 3964-3971.e7, doi:10.1016/J.CELREP.2020.02.111.

7. Bahl, K.; Senn, J.J.; Yuzhakov, O.; Bulychev, A.; Brito, L.A.; Hassett, K.J.; Laska, M.E.; Smith, M.; Almarsson, Ö.; Thompson, J.; et al. Preclinical and Clinical Demonstration of Immunogenicity by MRNA Vaccines against H10N8 and H7N9 Influenza Viruses. Molecular Therapy 2017, 25, 1327, doi:10.1016/J.YMTHE.2017.03.035.

8. Walt, D.R.; Ogata, A.F.; Cheng, C.-A.; Desjardins, M.; Senussi, Y.; Sherman, A.C.; Powell, M.; Novack, L.; Von, S.; Li, X.; et al. Circulating Severe Acute Respiratory Syndrome Coronavirus 2 (SARS-CoV-2) Vaccine Antigen Detected in the Plasma of MRNA-1273 Vaccine Recipients. Clinical Infectious Diseases 2021, doi:10.1093/CID/CIAB465.

9. Abramczyk, H.; Surmacki, J.M.; Brozek-Pluska, B.; Kopec, M. Revision of Commonly Accepted Warburg Mechanism of Cancer Development: Redox-Sensitive Mitochondrial Cytochromes in Breast and Brain Cancers by Raman Imaging. Cancers 2021, Vol. 13, Page 2599 2021, 13, 2599, doi:10.3390/CANCERS13112599.

10. Movasaghi, Z.; Rehman, S.; Rehman, I.U. Raman Spectroscopy of Biological Tissues. Applied Spectroscopy Reviewss 2007, 42, 493541, doi:10.1080/05704920701551530.

11. Abramczyk, H.; Brozek-Pluska, B.; Kopec, M. Double Face of Cytochrome c in Cancers by Raman Imaging. Scientific Reports 2022, accepted 2022.

12. Abramczyk, H.; Surmacki, J.M.; Brozek-Pluska, B.; Kopec, M. Revision of Commonly Accepted Warburg Mechanism of Cancer Development: Redox-Sensitive Mitochondrial Cytochromes in Breast and Brain Cancers by Raman Imaging. Cancers 2021, Vol. 13, Page 2599 2021, 13, 2599, doi:10.3390/CANCERS13112599.

13. Vaughn, A.E.; Deshmukh, M. Glucose Metabolism Inhibits Apoptosis in Neurons and Cancer Cells by Redox Inactivation of Cytochrome c. Nature cell biology 2008, 10, 1477, doi:10.1038/NCB1807.

14. Abramczyk, H.; Brozek-Pluska, B.; Kopec, M.; Surmacki, J.; Błaszczyk, M.; Radek, M. Redox Imbalance and Biochemical Changes in Cancer by Probing Redox-Sensitive Mitochondrial Cytochromes in Label-Free Visible Resonance Raman Imaging. Cancers 2021, 13, 960, doi:10.3390/CANCERS13050960.

15. Abramczyk, H.; Surmacki, J.M.; Brozek-Pluska, B. Redox State Changes of Mitochondrial Cytochromes in Brain and Breast Cancers by Raman Spectroscopy and Imaging. Journal of Molecular Structure 2022, 1252, doi:10.1016/j.molstruc.2021.132134.

16. Falschlehner, C.; Schaefer, U.; Walczak, H. Following TRAIL's Path in the Immune System. Immunology 2009, 127, 145-154, doi:10.1111/j.1365-2567.2009.03058.x.

17. Seder, R.A.; Paul, W.E. Acquisition of Lymphokine-Producing Phenotype by CD4+ T Cells. Annu Rev Immunol 1994, 12, 635673, doi:10.1146/annurev.iy.12.040194.003223.

18. Romagnani, S. Lymphokine Production by Human T Cells in Disease States. Annu Rev Immunol 1994, 12, 227-257, doi:10.1146/annurev.iy.12.040194.001303.

19. Unsay, J.D.; Cosentino, K.; Subburaj, Y.; García-Sáez, A.J. Cardiolipin Effects on Membrane Structure and Dynamics. Langmuir 2013, 29, 15878-15887, doi:10.1021/la402669z.

20. Molecular Expressions Cell https://micro.magnet.fsu.edu/cells/mitochondria/mitochondria.html (accessed on 13 January 2022).

21. Abramczyk, H.; Imiela, A.; Śliwińska, A. Novel Strategies of Raman Imaging for Exploring Cancer Lipid Reprogramming. Journal of Molecular Liquids 2019, 274, 52-59, doi:10.1016/j.molliq.2018.10.082. 
554 22. Abramczyk, H.; Imiela, A.; Surmacki, J. Novel Strategies of Raman Imaging for Monitoring Intracellular Retinoid Metabolism 555 in Cancer Cells. Journal of Molecular Liquids 2021, 334, 116033, doi:10.1016/j.molliq.2021.116033.

556 23. Suzuki, Y.J.; Gychka, S.G. SARS-CoV-2 Spike Protein Elicits Cell Signaling in Human Host Cells: Implications for Possible Consequences of COVID-19 Vaccines. Vaccines (Basel) 2021, 9, 36, doi:10.3390/vaccines9010036. 


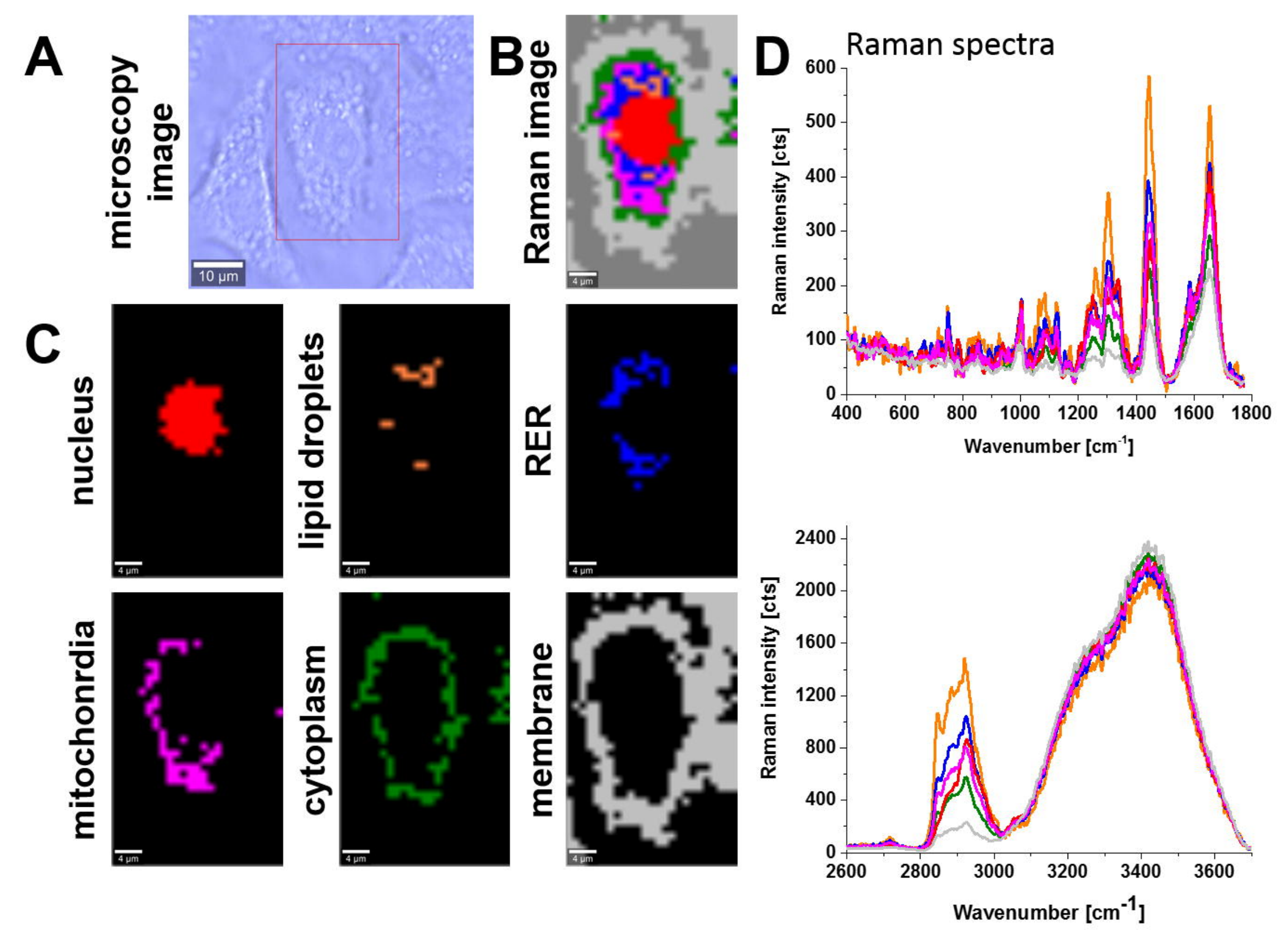



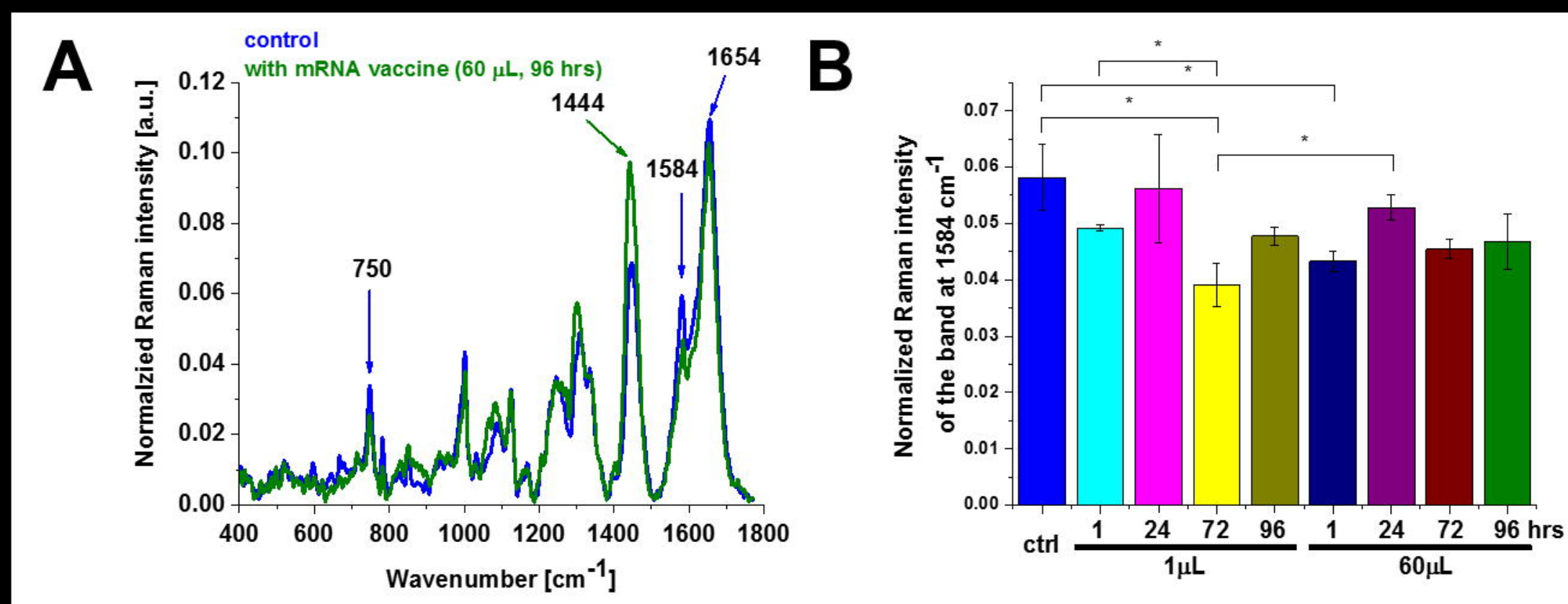

C
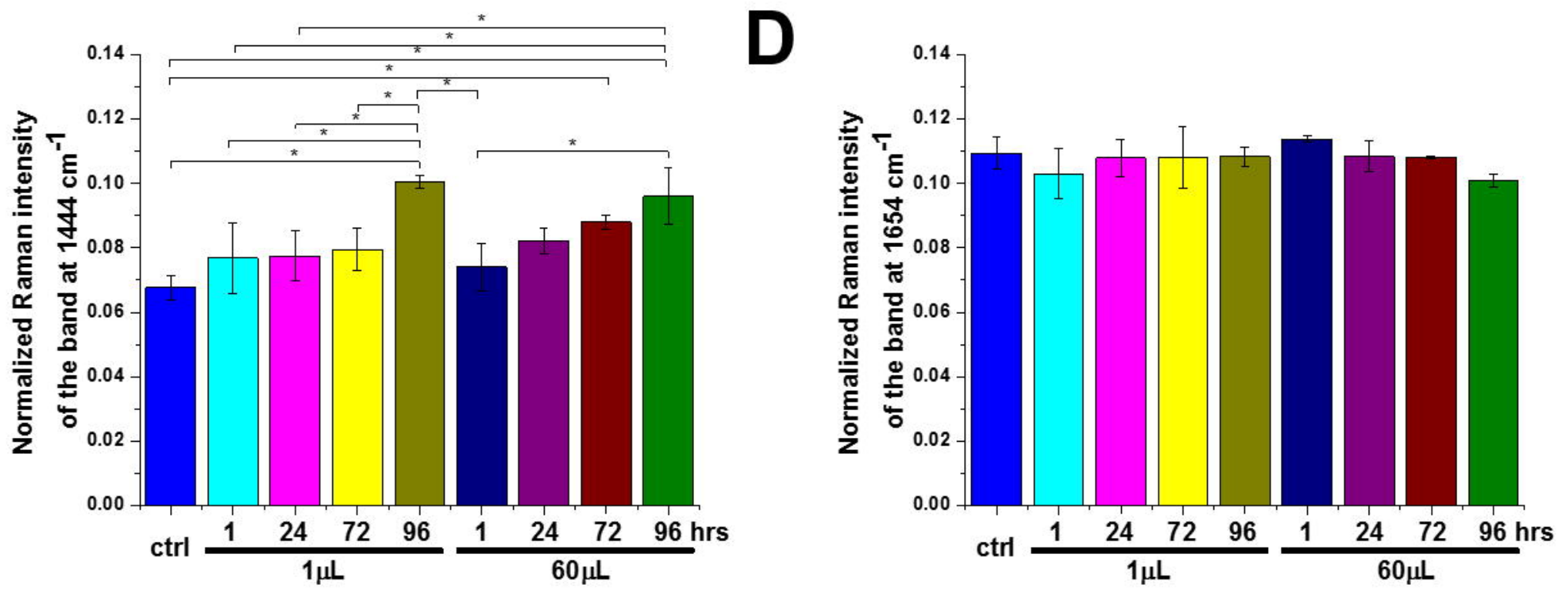

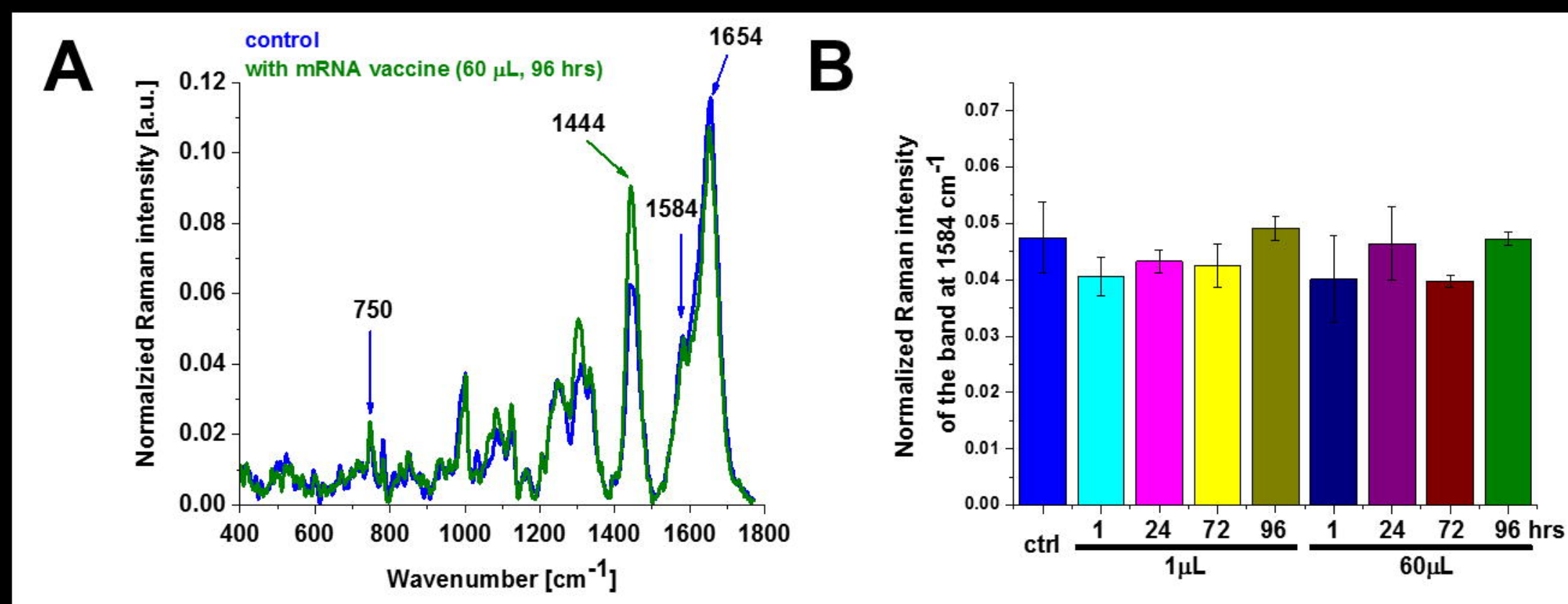

C
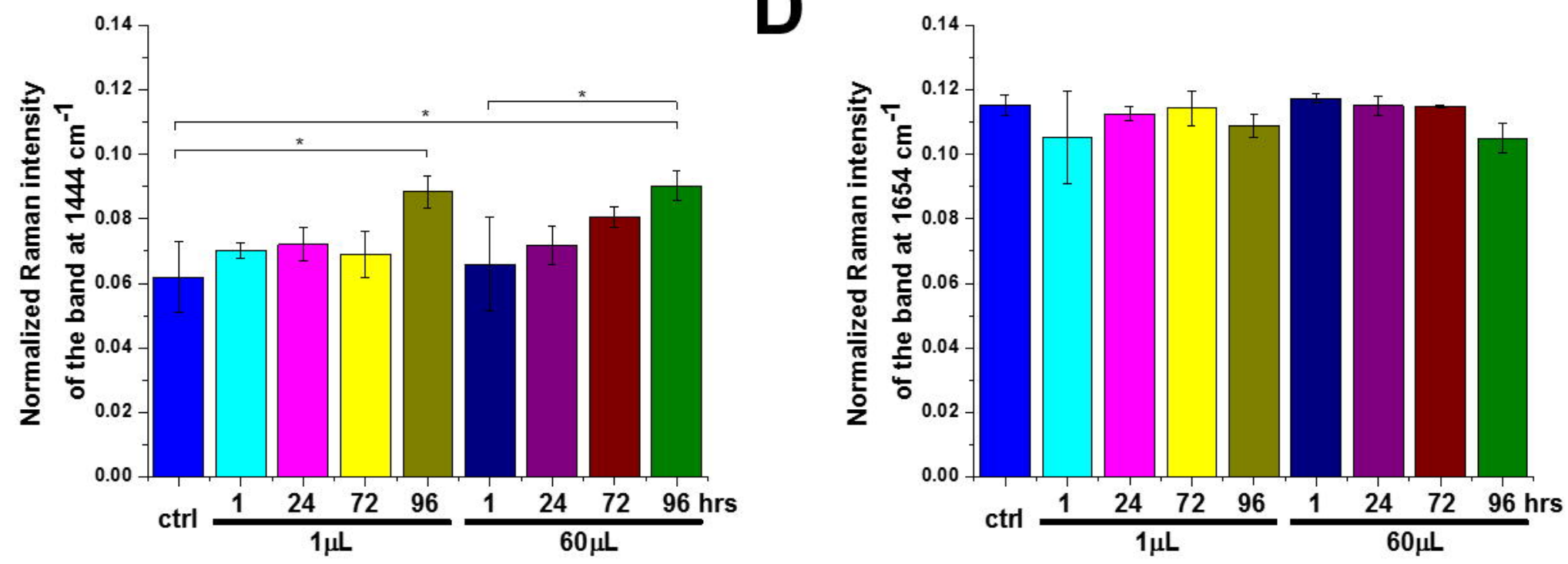

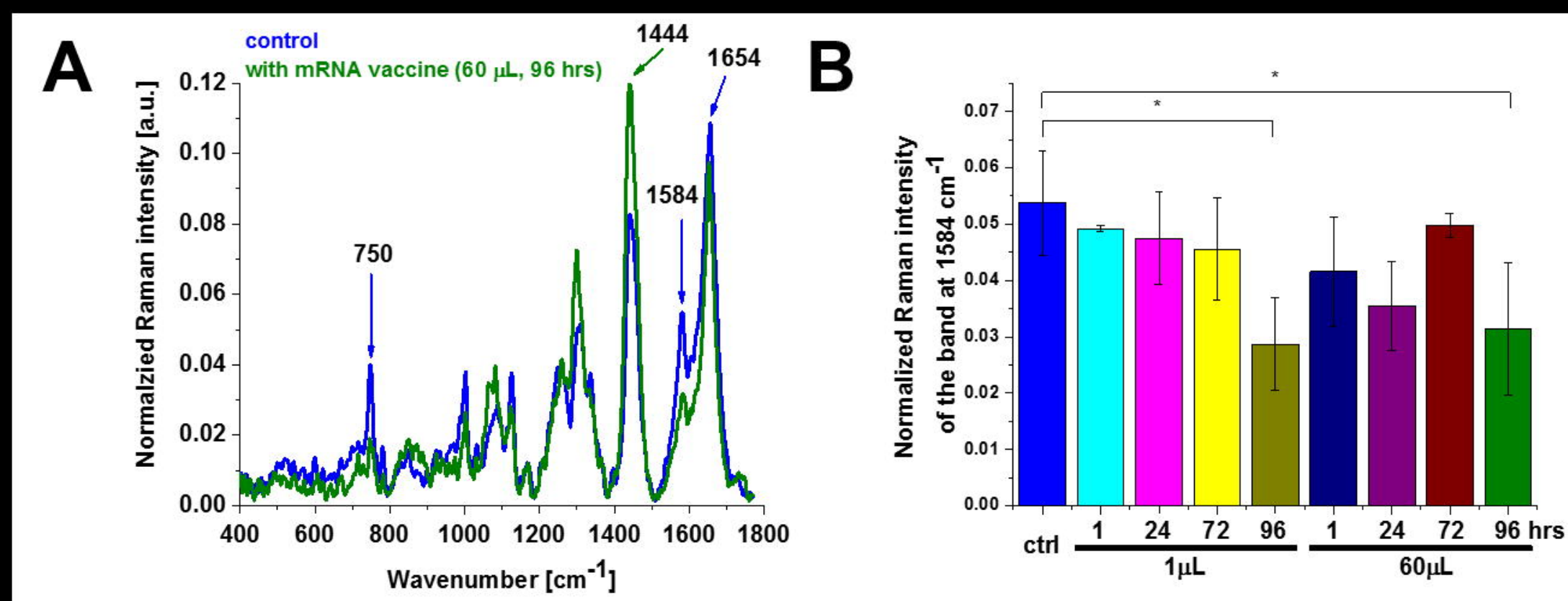

C
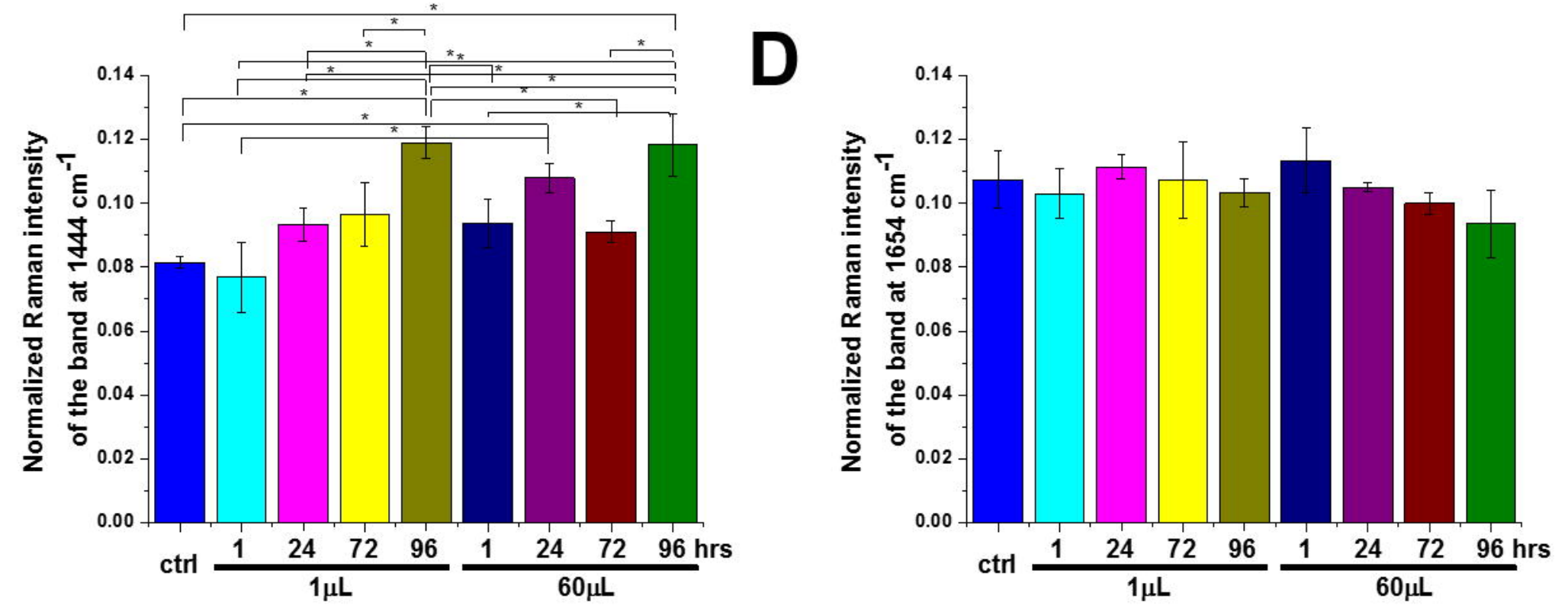

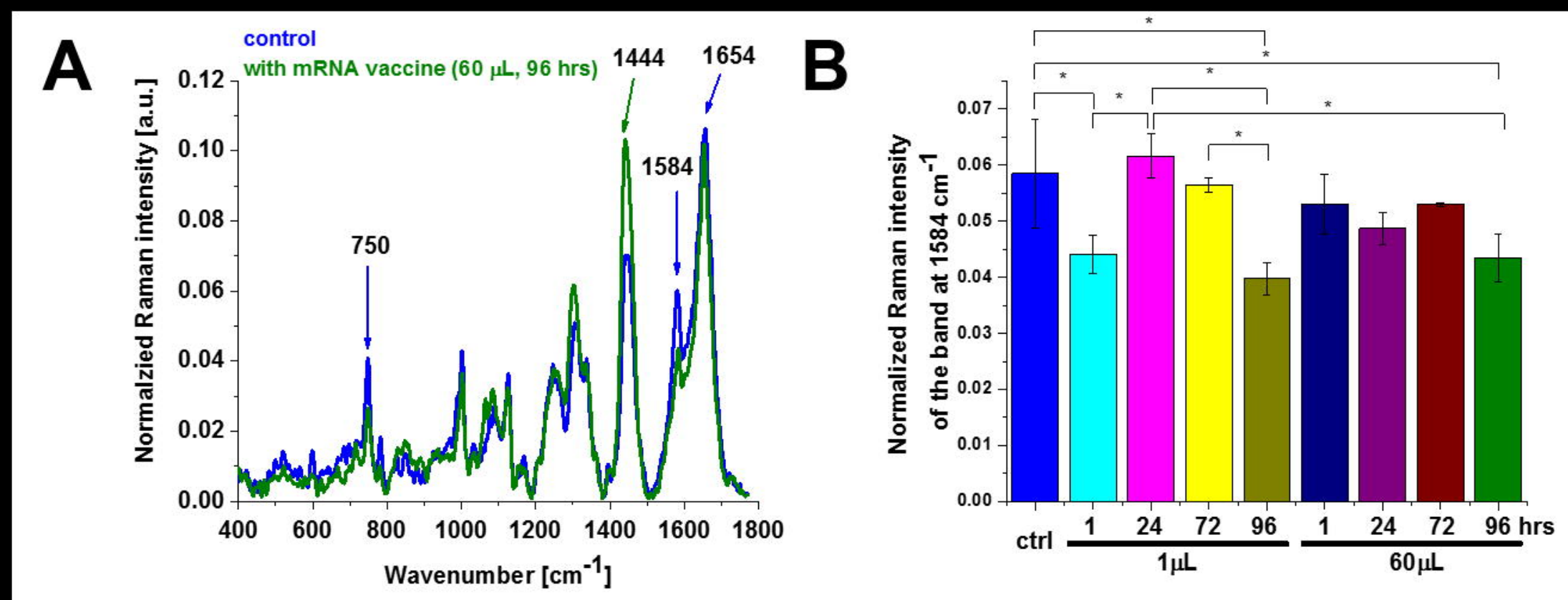

C
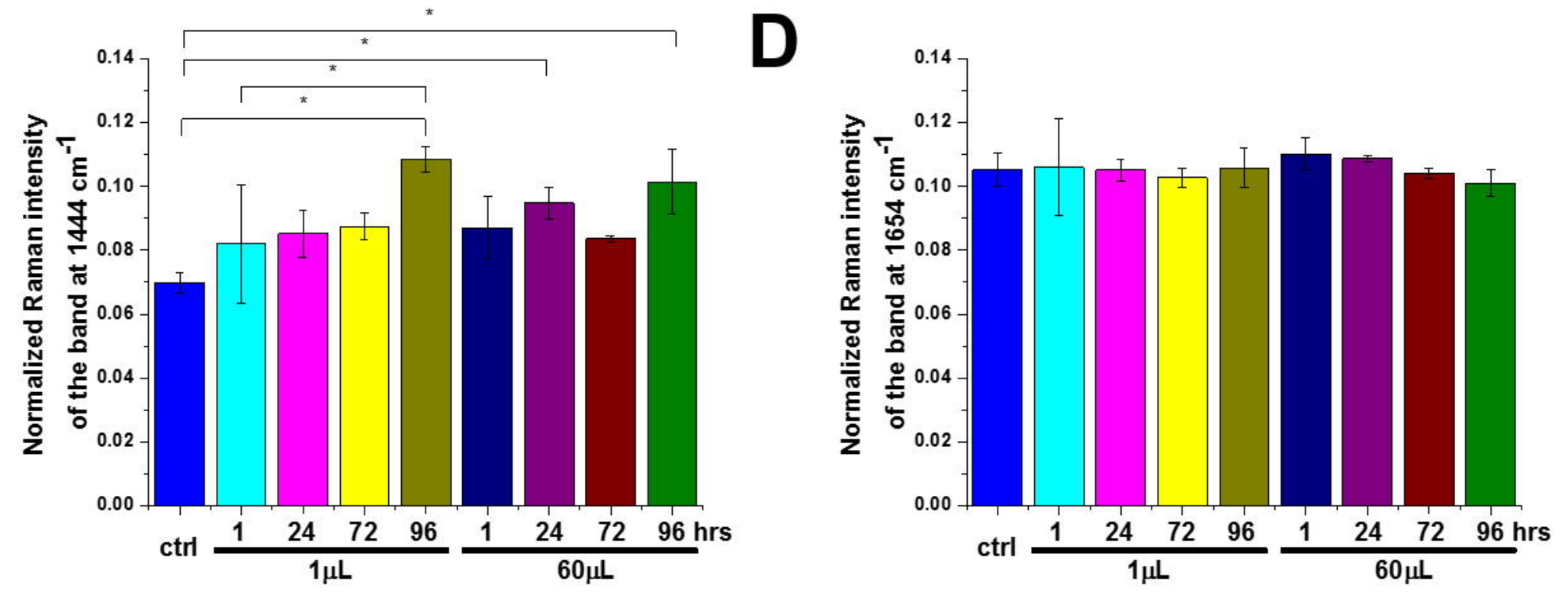

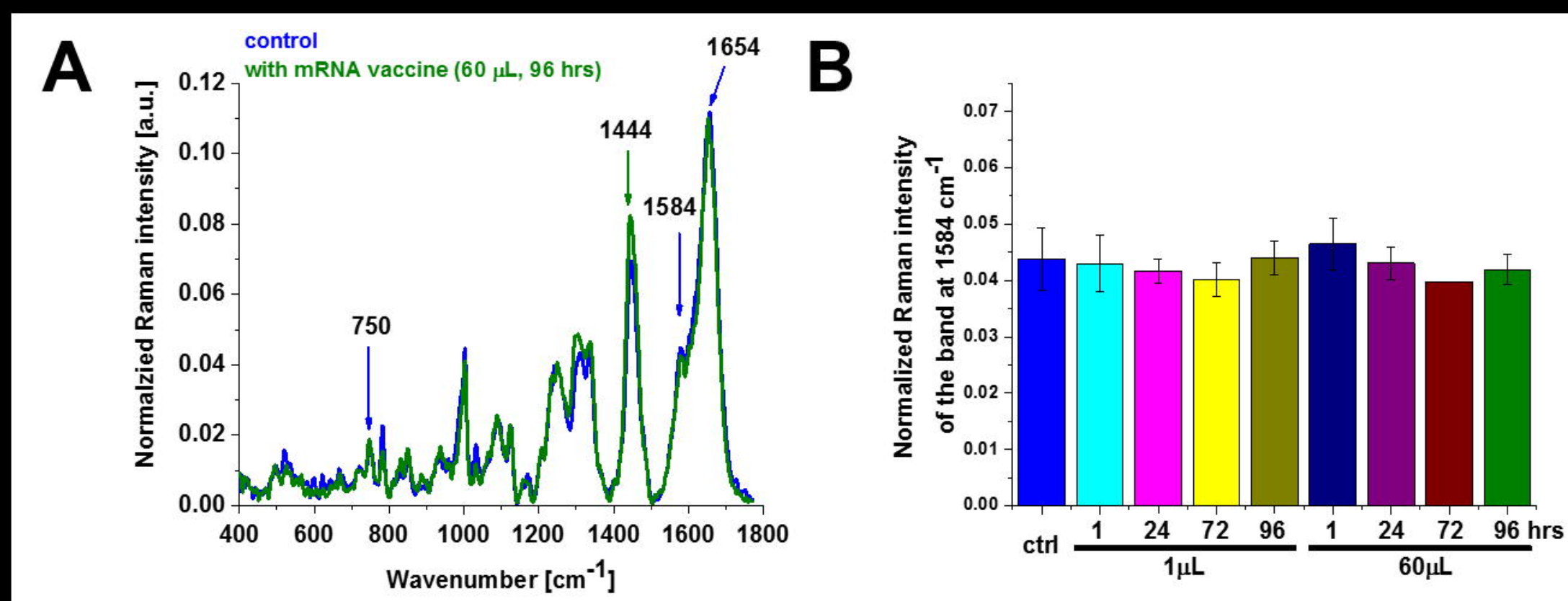

C
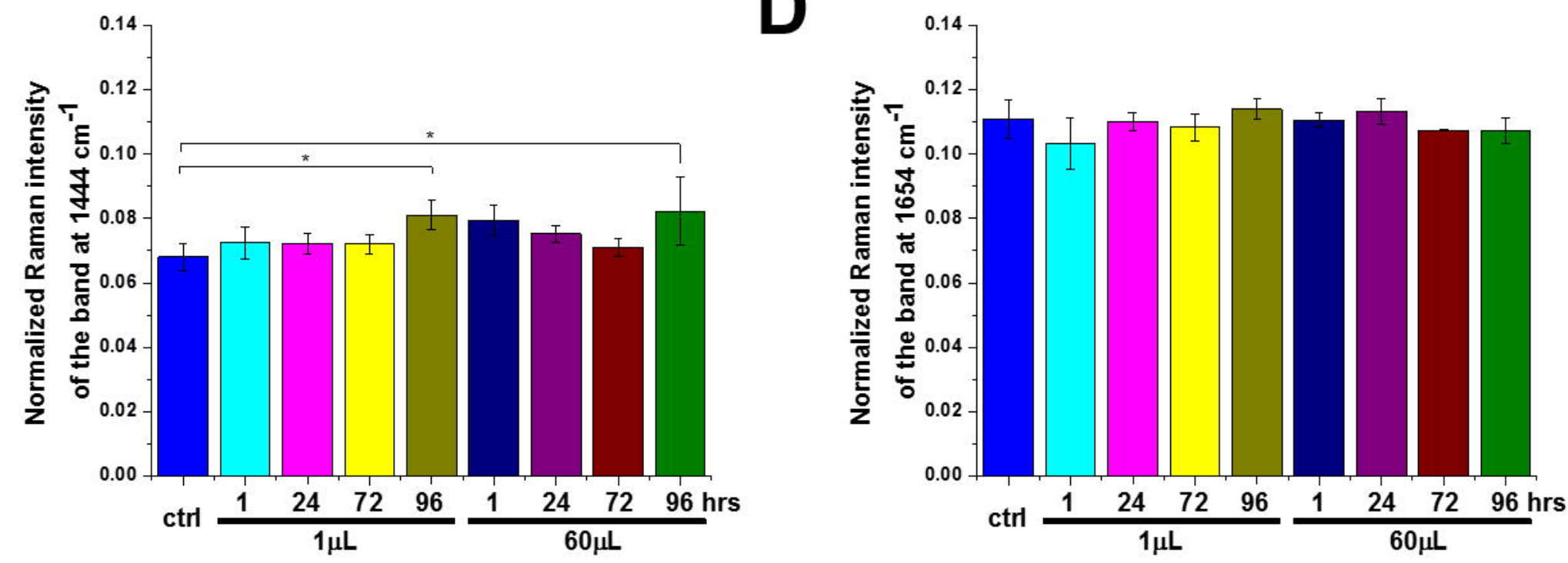

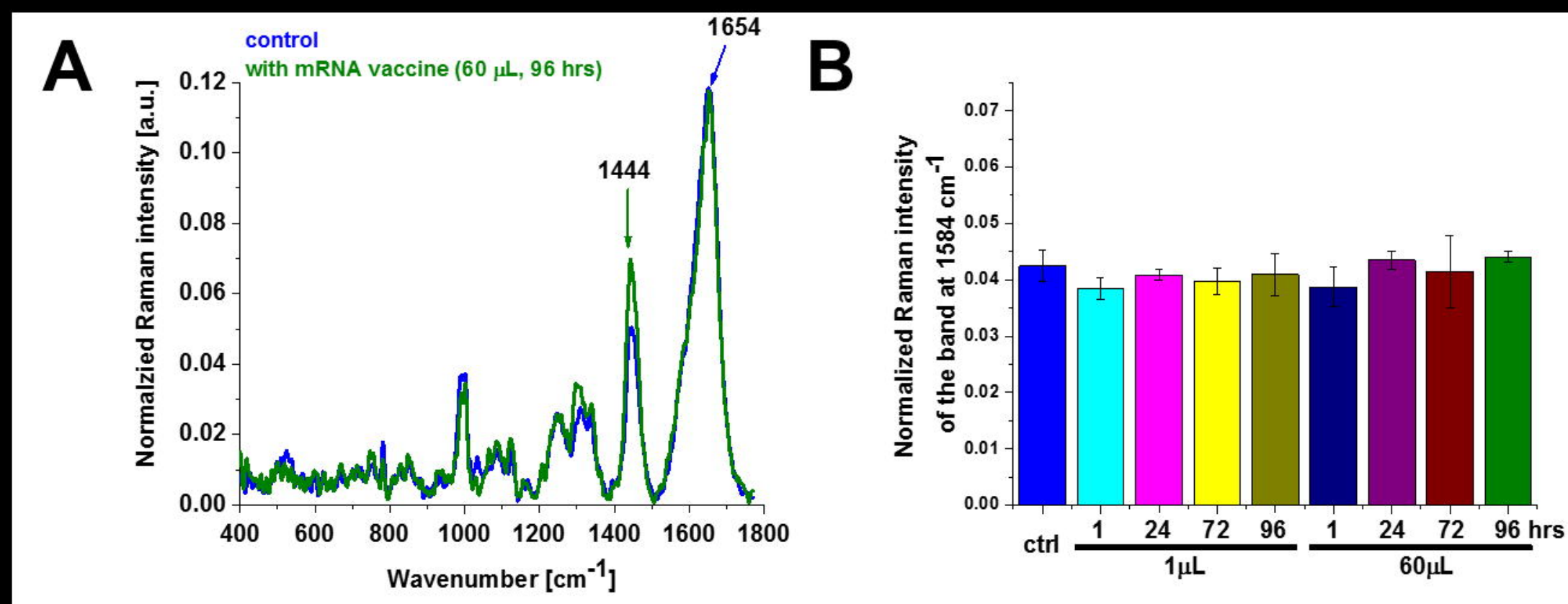

C
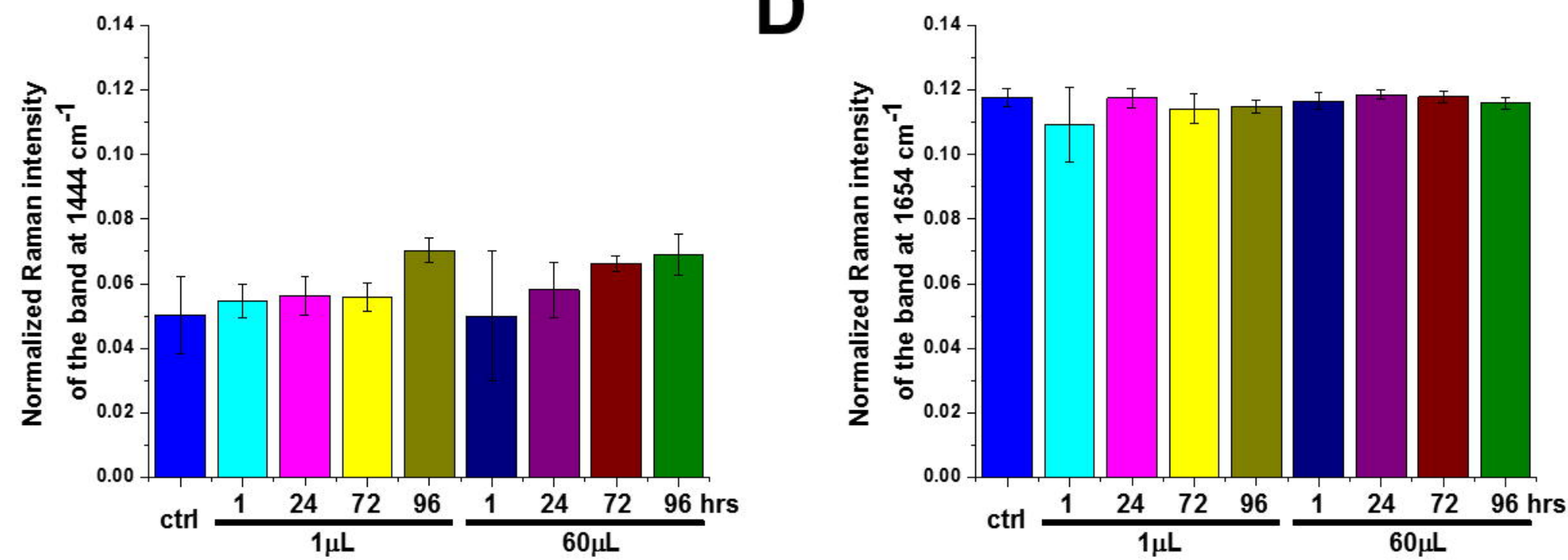


\section{virtual excited state}

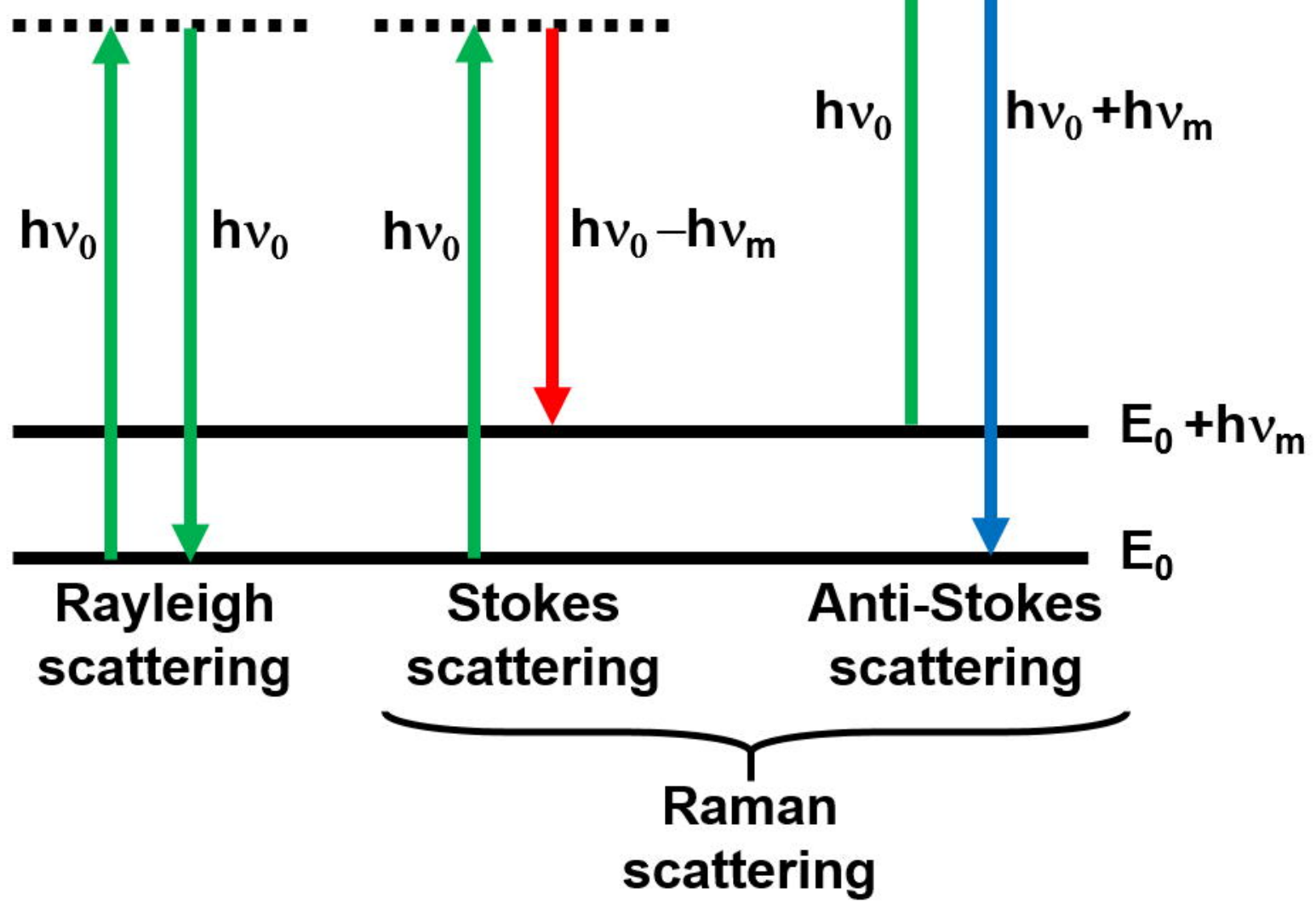




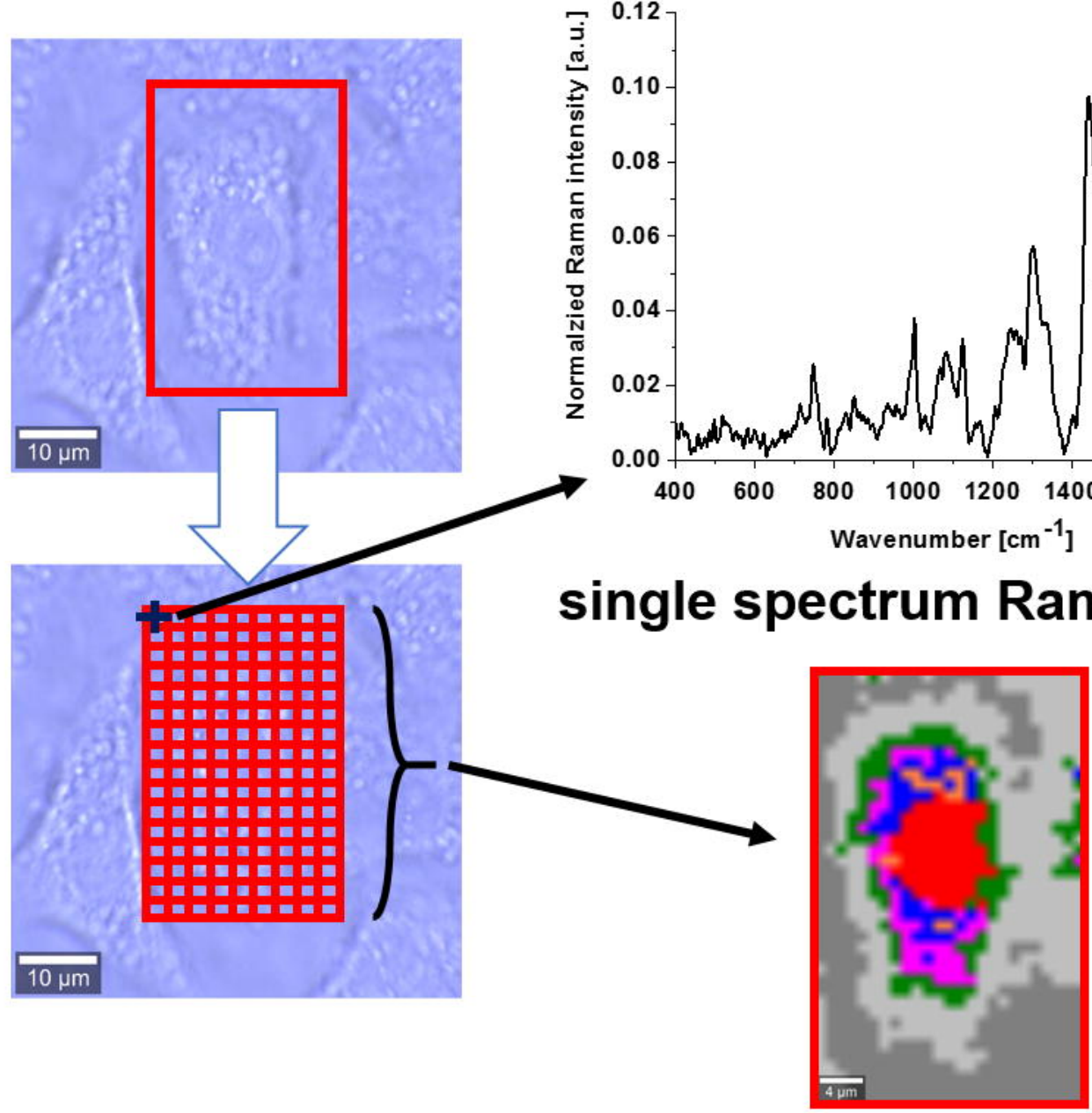

Raman imaging mode 